\title{
An energy-based interaction model for population opinion dynamics with topic coupling
}

\author{
Hossein Noorazar $^{* 1}$, Matthew J. Sottile ${ }^{\dagger 1}$, and Kevin R. Vixie ${ }^{\ddagger 1}$ \\ ${ }^{1}$ Department of Mathematics and Statistics, Washington State University
}

\begin{abstract}
We introduce a new, and quite general variational model for opinion dynamics based on pairwise interaction potentials and a range of opinion evolution protocols ranging from random interactions to global synchronous flows in the opinion state space. The model supports the concept of topic "coupling", allowing opinions held by individuals to be changed via indirect interaction with others on different subjects. Interaction topology is governed by a graph that determines interactions. Our model, which is really a family of variational models, has, as special cases, many of the previously established models for the opinion dynamics.

After introducing the model, we study the dynamics of the special case in which the potential is either a tent function or a constructed bell-like curve. We find that even in these relatively simple potential function examples there emerges interesting behavior. We also present results of preliminary numerical explorations of the behavior of the model to motivate questions that can be explored analytically.
\end{abstract}

Keywords:- Opinion Game, Opinion dynamics, Naming Game, Social Interaction

\section{Introduction}

An Opinion Game is a mathematical model of the evolution of the opinions of agents within a population, from creation of an idea to its diffusion through the population and finally the ultimate state of the system. Opinion games (or opinion dynamics models) have been studied since the middle of the 20th century. They often are used to study the dynamics of generic opinions that flow within a population.

By creating a formal mathematical model we can study detailed hypotheses about transmission mechanisms and the impact of population structure on opinion formation. Clearly such models are idealized and fail to capture all the nuances and fuzziness of individual

\footnotetext{
*hnoorazar@math.wsu.edu

†msottile@math.wsu.edu

${ }_{\ddagger}^{\ddagger}$ vixie@speakeasy.net
} 
human thought, but they give at least a disciplined glimpse into an aspect of how agents interact. A formal analytic approach also admits the ability to establish theorems about opinion dynamics, allowing one to derive conclusions with a rigorous chain of formal reasoning supporting them. This differs from approaches that take a purely simulation-based approach in which conclusions are derived from statistical properties of the resultant data instead of the underlying dynamical rules dictating interactions. By building a solid foundation for the opinion dynamics we get connection between model structure and statistical properties.

Models typically vary in the assumptions that they make about the nature of individual interactions and the overall goal of the population in the limit of an arbitrary number of interactions. In some cases, models are used to study specific classes of opinions that are created and flow within a population (such as linguistic games [3, 4, 25,37]). In other cases, the model seeks to explore equilibrium states in which not all agents necessarily agree, but reach opinion states that no longer change [16]. The generalized version of these games can be split into two categories. A binary (or more generally, discrete) opinion model [10,14,21, $22,31,41,42]$ requires agents to negotiate an opinion on a topic that has two possibilities and therefore, the final state of the system divides agents into two distinct groups, see [40], [5]. The alternative is a system in which the opinion space is continuous $[8,15,23,44]$. For example "How good do you think the New York Times is as a media outlet?" does not have a binary answer - you might strongly believe it is a strictly good or bad source of information, or you may have a mixed opinion somewhere in between. An opinion game model allows us to study the state reached after a set of individuals interacts an arbitrary number of times. This final state may be consensus or there may emerge stable sub-populations (clusters) in which members of each group reach consensus, but difference in opinion exist across these groups. The concept of opinion can be extended to higher dimensions, allowing for more than two extreme positions to be taken on a topic (e.g., "what is your favorite color?").

\subsection{Understanding social dynamics}

The dynamics of an opinion game are dictated by the micro-dynamics that occur in individualto-individual interactions between agents connected by some network topology. At the microscale we would represent the value of each opinion held by each individual, and how opinions of two individual can change and evolve via direct interaction. An example would be the one in which both agents have the same power over each other and therefore, after an interaction they would learn equally from each other and move relative to their interaction partner by the same amount either in the direction of increased agreement or disagreement or do not change whatsoever. For example, repulsion could occur when two agent's opinion are too far apart, especially when they talk about sensitive topics such as religion or politics. In some situations we may wish to model interactions where a difference of opinion between two individuals is insufficiently large to cause either attractive or repulsive behavior in which no change occurs. In yet another situation we may model dynamics in which opinions change only when they are sufficiently different in a repulsive fashion, but with no attractive behavior when they are near by. We will see how these can be modeled in section 3.4 via an interaction rule that seeks to minimize an interaction energy via potential functions. 


\subsubsection{Asymmetry of influence}

In the real world, it is frequently the case that agents do not have equivalent influence over each other. Such asymmetric relationships are commonplace: teacher/student, parent/child, expert/amateur, and so on. This can be modeled by applying weights, or influence power, to the relationship between agents. In this case the movements of the agents are not the same. For instance, if two agents are talking about chemistry and one is a chemist and the other is a student, then we can add an influence factor to the interaction and make the chemist to have more influence on the student (expert power). The result of such weighted influence would cause the student to experience significantly higher changes of opinion than the teacher during an interaction (which is part of the learning process), while allowing the teacher to admit some small level of change as a part of their interaction to maintain consistency in what they teach while allowing for some level of flexibility in response to their environment.

In an extreme case, we can consider the situation in which one agent is absolutely dominant and acts purely as a speaker and the other is acting purely as a hearer such that the opinion is transmitted unidirectionally. Consequently after an interaction, speaker would not change her opinion and hearer is the only one who changes. This allows for the modeling of information sources such as news or propaganda outlets that act in a purely influencing role within the population. It is worth mentioning that influence of agents over each other might change over time and evolve, we refer enthusiastic reader to [18]. Expert power is one mechanism by which asymmetry is introduced into an opinion model and is applied on the interaction between two individuals.

\subsubsection{Tendency for individualism}

The dynamics that emerge often fail to model phenomena that appear in real social systems due to decisions made solely by individuals. We can add more ingredients such as adaptive noise to agents' decisions to capture these decisions. This noise can be a function of the collective opinion within the population or cluster that an individual finds themselves a member of. For example, individuals that find themselves in a population that all hold the same opinions may have a desire to express their individuality by making an individual internal decision to change their opinion slightly to differentiate themselves. Exactly when and how this desire for individuality will emerge and balance with a desire to conform and form coherent like-minded groups is question open to investigation.

Different real world phenomena emerge due to this desire for individualism such as fads and trends, as well as the spontaneous emergence of new trends. In the fashion world, when a new style becomes popular there is a period in which individuals adapt their opinions about fashion towards this popular style. Eventually when this style becomes widespread and relatively commonplace, some individuals suddenly express a desire to be unique and begin avoiding this style. The more popular the style becomes, the more powerful would be tendency of peers to try something new and leave the cluster. This force is referred to as a "disintegrating force" or "tendency for individualism" or "tendency for uniqueness" [32]. Many formal models of opinion dynamics introduced in the 20th century are based on the assumption that such disintegrating forces are not present, which yields interesting but 
relatively synthetic idealized opinion dynamics and equilibria.

\subsubsection{Coupling of topics}

It is rarely the case that opinions on specific topics exist in isolation. An opinion about one topic frequently influences the opinion an individual holds on other topics. These range from the trivial (e.g., a preference for musical style influencing ones like or dislike of a given musician in a given genre), to those that reflect complex social influences (e.g., an opinion about religious belief influencing opinions about reproductive rights). Mathematically we can treat opinions that change together as being coupled, where there exists some functional that defines how the state of two or more coupled opinions co-evolve. In systems such as linguistic games to determine color terminology, coupling rarely has an impact, but to model social topics that have overlap at some level, coupling is an unavoidable aspect of capturing the complexity and nuance of human behavior. There are some works $[15,28,34]$ in which several topics are being discussed in the system, but the topics are independent and coupling is not present. A recent work [35] studies coupling of interdependent topics for the FriedkinJohnsen (FJ) model. A dynamical system view is presented in [34] along with an upper bound for convergence time of the Hegselmann-Krause model [6, 23].

\subsubsection{Interconnection topology}

Geographic and social factors dictate the likelihood of any two individuals interacting. This can be encoded via an interaction network. Given a population of individuals, the likelihood of any pair of individuals interacting is strongly influenced by their social and geographic network. Agents who are friends are more likely to interact than agents who are not, as are agents who are nearby versus those that are physically separated by a large distance. In our model, we impose a graph topology on all individuals who can interact in which the connectivity of the graph dictates which pairwise interactions are possible. For example, a tightly knit community of a small number of individuals likely can be treated as a fully connected graph in which all individuals may interact with each other. On the other hand, a set of individuals in widely distributed cities who do not interact with each other but read the same newspaper can be modeled as a star graph. Realistic interaction networks can be derived from social networking sites such as Twitter or Facebook, allowing for connectivity graphs that mimic interactions that are encountered in the real world.

\subsection{Contributions}

The model we present in this paper makes a number of noteworthy contributions to the study of opinion dynamics building upon decades of related research (Detailed in Sec. 2).

- The treatment of opinion evolution as a result of minimization of a (potentially nonlinear) energy functional based on the difference of opinion on a topic between interacting individuals. (Sec. 3.4)

- Demonstration that this model is a generalization of a family of previously published models from the literature by embedding them within the framework that we present. (Sec. 3.5.4) 
- Coupling of topics based on kernel-based coupling functions, to model the influence of explicit communication on one or more topics on a broader set of opinions held on topics not communicated. (Sec. 3.6)

- A study of the dynamical systems properties of our model to understand the existence of regions of the opinion space that represent basins of attraction, fixed points and sensitivity. (Sec. 4)

A set of results of computational experiments are provided demonstrating some of the dynamics one can observe in these models in addition to the analytical results that we derive. We close by posing a set of directions of potential future research based on our model and our early explorations with it.

\section{Related research}

Models of opinion dynamics have been studied over a long period of time with various formal methods and goals. In this section we provide a short review of noteworthy works in this history and discuss how our work is novel relative to prior work. This review is not intended to be exhaustive.

\subsection{French model: early formal modeling of opinion dynamics}

One of the earliest works in this area appears in 1956 [16], in which French considers agents as particles in a physical system which can induce forces on each other referred to as interagental power. Interagental power can be characterized in five ways: attraction power, expert power, reward power, legitimate power and coercive power. Let $v_{1}$ and $v_{2}$ be two agents in the network, then attraction power of $v_{1}$ over $v_{2}$ is how much $v_{2}$ likes $v_{1}$. Agents tend to listen to those whom they like and respect. Expert power is based on $v_{2}$ 's understanding of $v_{1}$ 's knowledge. The student/professor example is the case in which a student believes their teacher knows more on a given subject, and therefore is influenced by their teacher. Reward power is based on the right of an individual to offer tangible rewards of any kind to somebody for doing what is wanted or expected of them. For example, parent might give a gift to a child for getting a good GPA in a year, or a boss can promote one of their employees. A negative example of reward power could be taking away a child's toy for not performing well in school. Coercive power is based on $v_{1}$ 's ability to impose sanctions on $v_{2}$, it uses the threat of force to gain compliance from another. The idea is that someone is forced to do something that he/she does not desire to do. For instance in a court room the judge has utmost absolute power over a defendant, or a boss has power over their employee. And finally legitimate power is based on how much $v_{2}$ believes $v_{1}$ has a right to prescribe their opinion. It comes from an elected or appointed position of authority and is based on the social norm which requires agents to be obedient to those who hold superior positions social structure.

At a given time, each individual is forced by the cumulative force applied by all of their neighbors and their own resistance to move towards an equilibrium point where the resultant force is zero. The result is linear model that determines behavior of the system 
and individuals. In this model, the tendency of individuals is to move towards a weighted mean of their opinions at each step. French also explores the role of connectivity between individuals by applying the model on different types of directed graphs, where direction of an edge denotes a unidirectional ("speaks to") relation.

\subsection{DeGroot's averaging model and social influence evolution}

The model described by DeGroot [9] is a simplified version of the model we describe in this paper. The core concept of this model is that for a population of $n$ individuals, their set of opinions on one topic at any given point in time can be represented as a vector $\mathbf{y}^{(t)} \in \mathbb{R}^{n}$. An $n$-by- $n$ row-stochastic matrix of influence weights, $\mathbf{P}$, is provided in which the element $\mathbf{P}_{i j}$ corresponds to the amount of influence that individual $j$ has on individual $i$ when updating their opinion. The diagonal elements $\mathbf{P}_{i i}$ represent each individuals opinion of their own degree of influence within the population, which can be interpreted as their confidence in their own opinion relative to their peers. The update process is simply matrix-vector multiplication:

$$
\mathbf{y}^{(t+1)}=\mathbf{P} \mathbf{y}^{(t)}
$$

In this model, given a set of initial opinions the result will converge to the single weighted average of the initial opinions. As shown in Figure (1), for a full graph the convergence is very regular for all individuals. When a random graph is used, the system still ends at an average value, but the individual trajectory to this final value is more interesting (not necessarily monotonic). Our model subsumes this behavior, and supports behavior that DeGroot's model cannot capture. For example, DeGroot's model implies that all individuals will seek consensus - no matter how far apart the starting opinions are, they will come together. Our model captures the case where sufficiently different opinions on a topic may result in an increase in disagreement (e.g., polarization of opinions) in which the final outcome is not a single value but two subpopulations that reach two distinct opposing opinions. We illustrate this in our experimental results shown in Sec. 5. As is clear from Eq. (1), each node updates their opinion based on their own and their neighbors opinions. An extension model in which each agent incorporates their initial opinion throughout all iterations was developed by Friedkin and Johnsen [17, 19,20].

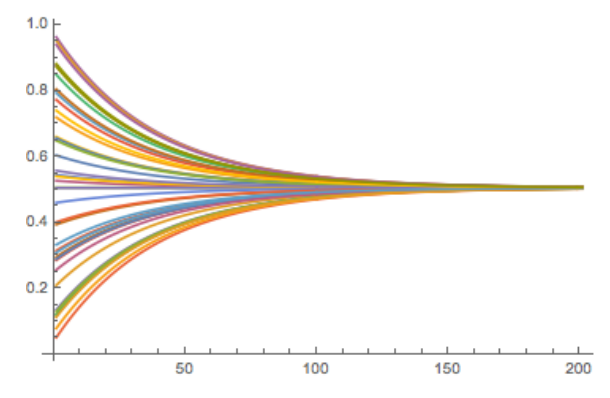

Figure 1: Evolution of the DeGroot model from a set of initial opinions distributed over $[0,1]$ to consensus. 
Moreover, an application of DeGroot's model, the DeGroot-Friedkin, model has recently been studied with respect to the evolution of social influence in work by Jia, et al. [24]. In French's model the influences are constant over time. In [24,30] authors model a new version of the game where influences of agents evolve depending on the outcome of the previous issue's result.

\subsection{The Bounded Confidence Model}

There are several different models for explaining and modeling the reasons and dynamics of phenomena such as the reason why agents tend to maintain their differences while they learn and become more alike by interacting. Why does the process of becoming more alike stop before a complete convergence to homogeneity of humans attributes? In 1997, Axelrod injected the idea of homophily in [2] to the opinion game which is referred to as "bounded confidence" model (BCM). In this model, individuals talk to agents who are similar to them and consequently become even more similar and agents whose opinions are too different do not interact. The pairwise interaction was developed later on by Deffuant [8] and a synchronized version is investigated by $[6,11,27,29]$. The influence of network topology on BCM dynamics is investigated in [43].

In this model at each time step a random pair of neighbors is chosen and the agents change their opinions if the difference of opinions are less than a threshold. BCM succeeded in explaining the formation of clusters, i.e. maintaining the differences between groups and not coming to a total consensus, to some extent, but it was fragile and sensitive. BCM generates opinion clustering in the context of discrete, categorical opinions rather than continuous opinions. Moreover, it is sensitive to "interaction noise" in the sense that even if with small probability agents talk to others whose opinions are not similar, i.e. talk to agents from different clusters, then we would see the emergence of a consensus monoculture or polarized final state. Therefore, BCM cannot fully explain coexistence of several different clusters in the system.

This is where Durkheim's theory [12] comes into play. Durkheim argues that the integrating forces that bind society together and binds an individual to others is that each individual adopts a linear combination of others' opinions. Mäs in [32] adopts Durkheim's theory and at a given time $t$, updates opinion of individual $i$ using weighted average of the difference between opinion of agent $i$ and the rest of population. In this manner, the system would not be sensitive to interaction noise and therefore tendency for individualism can be added to the system so that it would not hurt coexistence of several clusters in the society.

Krause [23] explains analytically and by simulations what can be said about the final state of a game given its initial profile for existing models in which opinion of each agent evolves as weighted average of other agents in the network. Different cases are considered where the weights are held constant or evolve over time. Negative ties, differentiation from and xenophobia toward those who are different, and its polarization effect is explained in $[1,13,36]$. Moreover, Anahita in [33] investigates the heterogeneous system, i.e the model in which each node has their own confidence interval. 


\subsection{Distributed behavior control and flocking models}

The set of opinions held by an individual on a set of topics can be thought of as nothing more than the state within that individual with respect to a set of variables. The association with these states and variables is largely an interpretation of this abstraction in order to study a specific system. Looking at other systems in which a set of agents with state interact, we can find interesting related work not originating in the study of opinion models in the context of distributed control problems in robotics. In [38] the problem of distributed behavior control in autonomous robots is investigated using a method similar to the energy potential model used in our model. This potential model for robotic control was introduced even earlier by Khatib [26] for obstacle avoidance purposes. In particular, the use of potential fields to model interactions removes the need for any centralized controller or state. Decision making can occur solely via pairwise interactions between robots and updates within each individual robot based on these pairwise interactions and knowledge about the interaction potential field. There is a striking similarity in the models of flocking behavior (e.g., boids [39]) to those in opinion dynamics in which a desire for population consensus (cohesion) is balanced with individual attraction or repulsion depending on proximity. Similar noise injection mechanisms to the social tendency towards individualism can also be found in flocking models to avoid having the system fall into a steady state. 


\section{Model Definition}

\section{Notation used throughout the paper}

- $\psi$ potential function.

- $\nabla \psi$ gradient of function $\psi$.

- $\tau$ parameter for some potential functions like (tip of the) tent, (bend point of) BCM potential.

- $\mathbb{O}$ opinion space.

- $\mathbb{T}$ topic space, if topic space is finite, $|\mathbb{T}|=n$.

- $o_{i}^{(t)}$ opinion of agent $i$ at time $t$ if there is only one topic in the system.

- $o_{i}^{(t)}\left(s_{k}\right)$ opinion of agent $i$ at time $t$ about topic $s_{k}$.

- $\dot{o}$ derivative of $o$ with respect to time.

- $\Delta o_{i}^{(t+1)}\left(s_{k}\right)=o_{i}^{(t+1)}\left(s_{k}\right)-o_{i}^{(t)}\left(s_{k}\right)$.

- $d_{i j}^{(t)}(s)=o_{i}^{(t)}(s)-o_{j}^{(t)}(s)$.

Difference between opinion of agents $i$ and $j$ about topic $s$ at a time $t$.
- $N(\mu, \sigma)$ normal distribution with mean $\mu$ and standard deviation $\sigma$.

- $\alpha$ learning rate.

- $\sigma(\mathbf{A})$ spectrum of matrix A.

- J Jacobian matrix.

- C coupling matrix.

- $c_{i j}$ inverse coupling strength of topic $i$ over topic $j$.

- $G=(V, E)$ graph $G$ with set of nodes $V$ and set of edges $E$.

- $n(i)$ set of neighbors of agent $i$.

- $\|$.$\| Euclidean norm.$

- vectors and matrices will be bold letters.

- $\mu_{s}$ mean of polarization counts

- $\mu_{p}$ mean of stabilization time

\subsection{Topic and opinion spaces}

First we will introduce definitions and associated notation that we will be using throughout this paper.

Definition 3.1. The set of topics being negotiated in the model constitute the topic space, denoted by $\mathbb{T}$. The topic space is effectively an index set, and for $n$ topics we can assume $\mathbb{T}=\mathbb{Z}_{n}$, and for an uncountable set of topics ordered on real line we have $\mathbb{T}:=[0, L]$.

Definition 3.2. The set of all possible (numerical) opinions, denoted by $\mathbb{O}$, is called opinion space, and will be a subset of an m-dimensional hypercube $\mathcal{C}^{m}$, for some $m$.

Remark 3.1. In this paper we focus on the case in which $\mathbb{O}=\mathcal{C}^{1}=[0,1]$ and this leads to the opinion state space $\mathbb{O}^{N}$ where $N$ is the population of the network.

For a given topic we assign an opinion value to it from the opinion space. For example if the question is "How good is the New York Times as a news source?" the opinion space 
would be $\mathbb{O}=[0,1]$. The interpretation of the endpoints is arbitrary, but we will adopt the convention when discussing opinions where zero corresponds to an absolutely negative opinion about a topic and one corresponds to an absolutely positive opinion. For more complex topics the opinion space is defined by either the $d-1$ dimensional simplex where $d$ corresponds to the number of extreme opinions that the topic supports and an opinion would be any point within the simplex, or d-dimensional hypercube where each dimension is a topic's attribute.

For example, if we look at the the color space defined by three primary colors - Red, Green and Blue - which is called the color triangle, then the color space (opinion space) would be a triangle and favorite color of an individual is a single point lying within the triangle. This opinion space can be naturally embedded inside a hypercube of dimension at least 3 .

On the other hand, we can take the RGB cube as our opinion space in which each dimension corresponds to a hue with the intensity of each hue varying between 0 and 1 . The favorite color of an individual is a vector in $\mathbb{R}^{3}$ lying inside the cube. The choice of specific opinion space used to encode specific topics is a problem dependent choice.

Definition 3.3. Opinion of an individual $i$ about topic $x$ at given time $t$ is denoted by $o_{i}^{(t)}(x) . o_{i}: \mathbb{T} \rightarrow \mathcal{C}^{m}$ is a function of both time and topic.

We may drop each of the subscripts/superscripts when the meaning is clear from the context.

\subsection{Interactions}

Individuals that interact to share and update their opinions are connected via an interaction network represented by a graph. Let $G=(V, E)$ represent the network under consideration where $V$ is the set of nodes and $E$ is the set of edges. The set of all neighbors of node $i \in V$ is denoted by $n(i)$. In this experimental results presented in this paper we only consider the fully connected network. One might consider different graphs such as star graph, ring, random graph, etc. and study the effect of graph measures, such as different centralities, on the opinion game.

Let $d_{i j}^{(t)}(x):=o_{i}^{(t)}(x)-o_{j}^{(t)}(x), \Delta o_{i}^{(t+1)}(x):=o_{i}^{(t+1)}(x)-o_{i}^{(t)}(x)$ for arbitrary topic $x$, and $w_{i j}$ to be the amount of influence of node $i$ on node $j . w_{j i}$ and $w_{i j}$ are not necessarily the same as the influence that two individuals have on each other is not necessarily symmetric (as in the case of a teacher/student or parent/child relationship).

Definition 3.4. Let $G=(V, E)$ be a given network. A sub-graph of $\mathrm{G}, \hat{G}=(\hat{V}, \hat{E})$, where $\hat{V} \subset V$ and $\hat{E} \subset E$, is called a $\epsilon$-cluster if $\forall i \in \hat{V} \exists j \in \hat{V}$ s.t. $\left|o_{i}-o_{j}\right|<\epsilon$.

\subsection{Units of time}

The model relates the state of opinions across the population to an abstract notion of time in order to study the evolution and dynamics of this opinion state. As such we must carefully define what constitutes a unit of time and how this choice has an impact on the evolution of 
the model. To start, we establish that there exists a maximum speed at which information can be propagated through the population in a single unit of time. In this model where all individuals are connected by an interaction network the minimum distance unit is a single edge in the network. We adopt the convention then that the maximum distance that information is allowed to flow is at most one edge from the origination point of the information.

A single time unit therefore allows any valid set of interactions such that this restriction on information flow distance holds. Formally, evolution of the system in a time unit entails a subset of individuals $\hat{V} \subset V$ and subset of edges representing pairwise interactions $\hat{E} \subset E$. The restriction on information flow holds when for the subgraph of $G, \hat{G}=(\hat{V}, \hat{E})$, there does not exist any path in $\hat{G}$ of length greater than 1 . Additional restrictions may also be imposed if interactions are directed (e.g., in the case of a hearer and speaker where only the hearer updates their state), such as the in-degree of all vertices in $\hat{V}$ being restricted to at most 1.

\subsection{Interaction energy potentials}

In our model the strength of an interaction is dictated by an interaction potential function $\psi$. While, in this paper we focus on those potentials which depend only on differences of opinions, our model does not require this in general. As a result, our model has most of the previous models in opinion dynamics as special cases. This is illustrated in 3.5.4 where we show that the Bounded Confidence model, the DeGroot model and the French model are special cases of our model.

Examples of potential function are illustrated in Fig. (2). The potential function determines the degree to which individuals must react in adjusting their own opinion depending upon the difference of their opinion $d_{i j}^{(t)}(x)$ when interacting with another individual on topic $x$. The interaction rules that update opinions of individuals encode this minimization by pushing individual opinions in the opposite direction of the gradient of the interaction potential $(-\nabla \psi)$. For example in the BCM, which is a special case of our model, individuals would learn from each other when their difference is less than a threshold and they do not interact otherwise. This potential function is illustrated in Fig. (2d) with a threshold of $\tau$. The tent function illustrated in Fig. (2a) represents the case where the individuals whose opinions difference is less than $\tau$ attract each other by becoming closer and repel by becoming further apart if their difference is more than $\tau$. In the case of the skewed flat top tent potential, shown in Fig. (2b), if the difference is between $\tau_{l}$ and $\tau_{r}$ then individuals are indifferent and will not change their position. The flat top tent potential is similar to that of the bounded confidence model, except that it not only forces individuals to come closer together when they are sufficiently similar but also causes them to repel when they are sufficiently different.

Given a pairwise update rule based on a potential function that dictates the attractive or repulsive relationship between two opinions, we must define an update rule for the entire population. Two options exist: one in which each step of the model involves only one pair of individuals, and one in which a maximal cohort is updated under a constraint dictated by the interaction network such that no individual makes more than one opinion update in a single step. In all cases the restrictions on information flow distance discussed in Sec. 3.3 


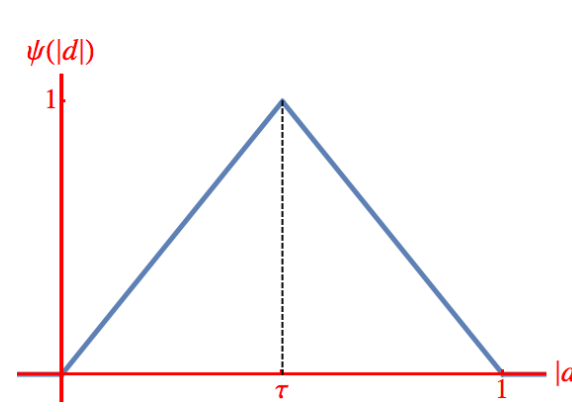

(a) Tent Potential

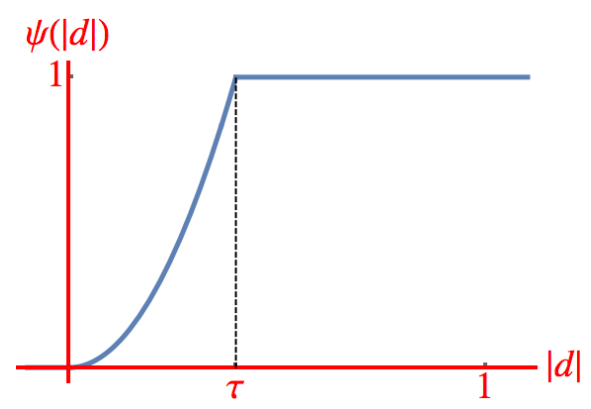

(d) BCM Potential

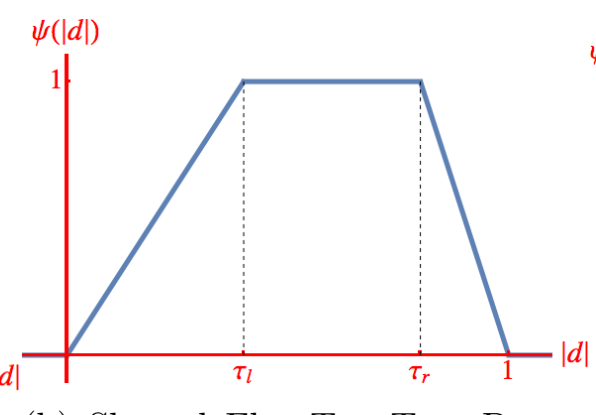

(b) Skewed Flat Top Tent Potential

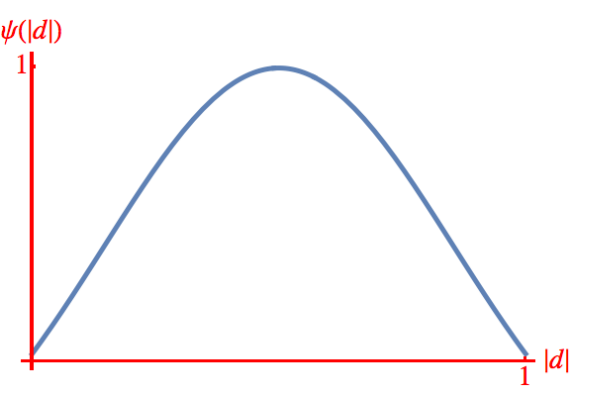

(c) Gaussian Potential

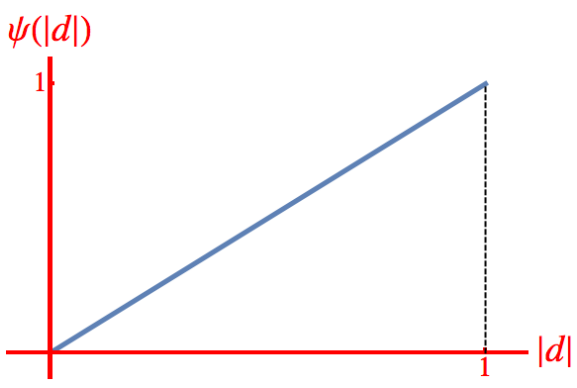

(e) A Simple Potential

Figure 2: Potential function examples

must be obeyed.

We will also discuss dynamics of the continuous game where the time is continuous and agents are continuously interacting.

\subsection{Individual and population update rules}

The model proceeds as a sequence of update steps in which one or more individuals update their opinion state based on a set of interactions. Update rules can be either applied oneat-a-time, in which random pairs of individuals interact, or in a concurrent population wide fashion. We start with the simplest case and build the model up. The continuous game updates, of course, are done synchronously, like a physical system with positively or negatively charged particles that are within reach of each other and exert force on one another.

\subsubsection{One-at-a-time updates - Derivation of interaction update rule}

We let pairs of individuals interact and update their opinions. Suppose there is one topic, $x$, in the system and two individuals $i$ and $j$ are interacting at a time $t$. Let $\psi: \mathbb{R} \rightarrow \mathbb{R}$ be a bounded potential energy function which measures the energy in the opinion interaction:

$$
Q_{i j}:=\psi\left(\left|d_{i j}\right|\right)=\psi\left(\left|o_{i}-o_{j}\right|\right)
$$

In order to reduce the energy of interaction, we have to move in the opposite direction of gradient of the energy function: 


$$
\frac{\Delta d_{i j}}{\Delta t}=-\alpha \frac{\partial}{\partial d} \psi(|d|)
$$

where $\alpha$ is a parameter that increases/decreases rate of change of opinions. We refer to $\alpha$ as the "learning rate". This would give us the update rule as follows:

$$
\frac{\Delta d_{i j}}{\Delta t}=\frac{d_{i j}^{(t+1)}-d_{i j}^{(t)}}{1}=-\alpha \psi^{\prime}\left(\left|d_{i j}\right|\right) \frac{d_{i j}^{(t)}}{\left|d_{i j}^{(t)}\right|}
$$

consequently,

$$
d_{i j}^{(t+1)}=d_{i j}^{(t)}-\alpha \psi^{\prime}\left(\left|d_{i j}\right|\right) \frac{d_{i j}^{(t)}}{\left|d_{i j}^{(t)}\right|}
$$

If we want to nudge both agents' opinion by the same amount we must have:

$$
\left\{\begin{array}{l}
o_{i}^{(t+1)}-o_{i}^{(t)}=-\frac{\alpha}{2} \psi^{\prime}\left(\left|d_{i j}^{(t)}\right|\right) \frac{d_{i j}^{(t)}}{\left|d_{i j}^{(t)}\right|} \\
-\left(o_{j}^{(t+1)}-o_{j}^{(t)}\right)=-\frac{\alpha}{2} \psi^{\prime}\left(\left|d_{i j}^{(t)}\right|\right) \frac{d_{i j}^{(t)}}{\left|d_{i j}^{(t)}\right|}
\end{array}\right.
$$

And therefore the update rule from time $t$ to $t+1$ is given by,

$$
\left\{\begin{array}{l}
o_{i}^{(t+1)}=o_{i}^{(t)}-\frac{\alpha}{2} \psi^{\prime}\left(\left|d_{i j}^{(t)}\right|\right) \frac{d_{i j}^{(t)}}{\left|d_{i j}^{(t)}\right|} \\
o_{j}^{(t+1)}=o_{j}^{(t)}+\frac{\alpha}{2} \psi^{\prime}\left(\left|d_{i j}^{(t)}\right|\right) \frac{d_{i j}^{(t)}}{\left|d_{i j}^{(t)}\right|}
\end{array}\right.
$$

If the two agents have different influences on each other, then the opinions change by different amounts. We can dictate this fact by inserting the influence weights into Eq.(7) as follows:

$$
\left\{\begin{array}{l}
o_{i}^{(t+1)}=o_{i}^{(t)}-\frac{\alpha}{2} w_{j i} \psi^{\prime}\left(\left|d_{i j}^{(t)}\right|\right) \frac{d_{i j}^{(t)}}{\left|d_{i j}^{(t)}\right|} \\
o_{j}^{(t+1)}=o_{j}^{(t)}+\frac{\alpha}{2} w_{i j} \psi^{\prime}\left(\left|d_{i j}^{(t)}\right|\right) \frac{d_{i j}^{(t)}}{\left|d_{i j}^{(t)}\right|}
\end{array}\right.
$$

Note that $o_{i}^{(t)}(x)$ is function of both time and the topic. So, if there are finite number of topics in the system, then the total energy between the two given individuals $i$ and $j$ would be the sum of all energies of opinion interactions. The tendency for individualism could be added to the model as well at this point. We will use the noise model introduced in [32]. By adding this term to Eq. (8), we get:

$$
\left\{\begin{array}{l}
o_{i}^{(t+1)}=o_{i}^{(t)}-\frac{\alpha}{2} w_{j i} \psi^{\prime}\left(\left|d_{i j}^{(t)}\right|\right) \frac{d_{i j}^{(t)}}{\left|d_{i j}^{(t)}\right|}+\xi_{i}(t) \\
o_{j}^{(t+1)}=o_{j}^{(t)}+\frac{\alpha}{2} w_{i j} \psi^{\prime}\left(\left|d_{i j}^{(t)}\right|\right) \frac{d_{i j}^{(t)}}{\left|d_{i j}^{(t)}\right|}+\xi_{j}(t)
\end{array}\right.
$$


where $\xi_{i}(t)$ is a random (sample) value from a distribution $\xi_{i}(t) \sim N\left(0, \sigma_{i}(t)\right)$ associated with individual $i$ at time $t$ with mean zero and variance defined by:

$$
\sigma_{i}(t)=\frac{s}{e-1}\left(-|N(i)|+\sum_{j \in N(i)} e^{1-\left|d_{i j}(t)\right|}\right)
$$

where the parameter $s$ is used to manipulate the strength of disintegrating forces. This tendency for uniqueness increases when the $d_{i j}$ 's are small, i.e. there is high uniformity.

If $o_{i}^{(t+1)}$ becomes more than 1 or less than zero we should set it to 1 or zero, respectively. This can be achieved by applying a clamping function:

$$
\operatorname{clamp}(x)= \begin{cases}0 & \text { if } x<0 \\ x & \text { if } 0 \leq x \leq 1 \\ 1 & \text { if } x>1\end{cases}
$$

\subsubsection{All neighbor interaction}

At this point we have derived a rule for updating opinions after a single interaction. Now we can use it for the model in which at each time step, each agent updates their opinion according to all of their neighbors' opinion, i.e. at each time step a agent would have interaction with all of their neighbors, and their opinion in time $t+1$ depends on all of her neighbor's opinion in time $t$. Let the node $i$ have $k$ neighbors, then the updating rule is given by:

$$
o_{i}^{(t+1)}=o_{i}^{(t)}+\sum_{j=1}^{k}-\frac{\alpha}{2} \psi^{\prime}\left(\left|d_{i j}\right|\right) \frac{d_{i j}}{\left|d_{i j}\right|}
$$

The influence weights can be added accordingly. The influence weights that individual $i$ assigns to their neighbors and themselves (resistance force) has to add up to 1 , so the weight matrix $w$ is stochastic:

$$
\sum_{j \in N(i)} w_{j i}=1
$$

This is the simplest case in which there is only one potential function for all interaction. We can also have a potential function for each pair or each agent could have her own potential function.

\subsubsection{Bidirectional vs unidirectional information exchange}

In the previous sections we assume that information flow is bi-directional in an interaction such that both individuals share their opinion state and both update it based on what they receive. It is also possible to model interactions as uni-directional such that there is a well defined speaker that shares information to a receiving hearer but not vice versa. This distinction can be encoded in the network through the use of directed versus undirected edges. It must be noted that a single undirected edge between individuals $i$ and $j$ is not 
equivalent to two directed vertices in opposing directions ( $i$ to $j$ and $j$ to $i$ ), as each edge corresponds to a single communication event.

A similar effect can be accomplished with undirected graphs through the use of zero weights. An individual $i$ that influences others will have weights $w_{i *}$ that are not necessarily zero, but if $i$ is not influenced at all by others then $\forall j \neq i, w_{j i}=0$. The use of weights in this manner extends to graphless models in which connectivity is based on spatial proximity where no notion of directedness or edge connectivity exists. We do not cover this mechanism for individual connectivity in this paper.

\subsubsection{Embedding other models into our model}

To show BCM is special case of our model, let the potential function be

$$
\psi(x)= \begin{cases}x^{2} & 0 \leq x \leq \tau \\ \tau^{2} & \tau<x \leq 1\end{cases}
$$

Then we have

$$
\psi^{\prime}(x)= \begin{cases}2 x & 0 \leq x \leq \tau \\ 0 & \tau<x \leq 1\end{cases}
$$

Hence, using (7) if the two agents are within confidence interval of each other we get:

$$
\left\{\begin{array}{l}
o_{i}^{(t+1)}=o_{i}^{(t)}-\frac{\alpha}{2}\left(2\left|d_{i j}\right|\right) \frac{d_{i j}^{(t)}}{\left|d_{i j}^{(t)}\right|}=o_{i}^{(t)}-\alpha d_{i j}^{(t)} \\
o_{j}^{(t+1)}=o_{j}^{(t)}+\frac{\alpha}{2}\left(2\left|d_{i j}\right|\right) \frac{d_{i j}^{(t)}}{\left|d_{i j}^{(t)}\right|}=o_{j}^{(t)}+\alpha d_{i j}^{(t)}
\end{array}\right.
$$

which is identical to the equation in section 2.1 of [8]. This is the pairwise interaction and it is easy to build the synchronized version.

We saw in the DeGroot model we have $\mathbf{y}^{(t+1)}=\mathbf{P y}^{(\mathbf{t})}$. The following conditions will show the DeGroot model is a special case of our model. Consider synchronized version of the game with the following conditions. Define potentials

$$
\psi_{i j}=\psi\left(o_{i}, o_{j}\right)= \begin{cases}-p_{i j} o_{i} o_{j} & j \neq i \\ -\frac{1}{2}\left(p_{i i}-1\right) o_{i}^{2} & j=i\end{cases}
$$

then,

$$
\frac{\partial}{o_{i}} \psi_{i j}= \begin{cases}-p_{i j} o_{j} & j \neq i \\ -\left(p_{i i}-1\right) o_{i} & j=i\end{cases}
$$

Now, the DeGroot model says $\mathbf{y}^{(t+1)}=\mathbf{P y}^{(t)}$ which is the same as $\mathbf{y}^{(t+1)}-\mathbf{y}^{(t)}=\left(\mathbf{P}-\mathbf{I}_{N}\right) \mathbf{y}^{(t)}$ where $\mathbf{I}_{N}$ is identity matrix of size $N$. Since we are letting self interaction, defined by $\psi_{i i}$ for individual $i$ we have the following, whose right hand side will still give us a gradient descent method: (Let $\alpha=2$ ) 


$$
\begin{aligned}
o_{i}^{(t+1)}-o_{i}^{(t)} & =-\psi_{i 1}^{\prime}\left(o_{i}^{(t)}, o_{1}^{(t)}\right)-\psi_{i 2}^{\prime}\left(o_{i}^{(t)}, o_{2}^{(t)}\right)-\cdots-\psi_{i i}^{\prime}\left(o_{i}^{(t)}, o_{i}^{(t)}\right)-\cdots-\psi_{i N}^{\prime}\left(o_{i}^{(t)}, o_{N}^{(t)}\right) \\
& =p_{i 1} o_{1}^{(t)}+p_{i 2} o_{2}^{(t)}+\cdots+\left(p_{i i}-1\right) o_{i}^{(t)}+\cdots p_{i N} o_{N}^{(t)}
\end{aligned}
$$

and therefore,

$$
\begin{aligned}
o_{i}^{(t+1)} & =-\psi_{i 1}^{\prime}\left(o_{i}^{(t)}, o_{1}^{(t)}\right)-\psi_{i 2}^{\prime}\left(o_{i}^{(t)}, o_{2}^{(t)}\right)-\cdots-\psi_{i i}^{\prime}\left(o_{i}^{(t)}, o_{i}^{(t)}\right)-\cdots-\psi_{i N}^{\prime}\left(o_{i}^{(t)}, o_{N}^{(t)}\right) \\
& =p_{i 1} o_{1}^{(t)}+p_{i 2} o_{2}^{(t)}+\cdots+p_{i i} o_{i}^{(t)}+\cdots p_{i N} o_{N}^{(t)}
\end{aligned}
$$

which is the $i^{t h}$ row of the DeGroot model matrix.

The interesting dynamics of French model [16] which works with digraphs comes from the topology of the network . Let $i$ be a node with indegree $k$ and define $N(i)=\left\{i_{1}, i_{2}, \cdots, i_{k}\right\}$ be the neighbors of agent $i$ who have power over it. Define the potential functions

$$
\psi_{i j}=\psi\left(o_{i}, o_{i_{j}}\right)= \begin{cases}-\frac{o_{i} o_{i_{j}}}{k+1} & 1 \leq i_{j} \leq k \\ \frac{k}{2(k+1)} o_{i}^{2} & i_{j}=i\end{cases}
$$

where $o_{i_{j}}^{(t)}$ is opinion of $j^{\text {th }}$ neighbor of $i$ which has power over $i$, i.e. there is an incoming edge from $i_{j}$ agent to $i$, and the case $i_{j}=i$ is the self-resistance element of French model, then we have

$$
\frac{\partial}{\partial o_{i}} \psi_{i j}= \begin{cases}-\frac{o_{i j}}{k+1} & 1 \leq i_{j} \leq k \\ \frac{k}{(k+1)} o_{i} & i_{j}=i\end{cases}
$$

and therefore, moving in the opposite of gradient of the potential function with synchronous update gives us the following step size

$$
o_{i}^{(t+1)}-o_{i}^{(t)}=\frac{1}{k+1}\left(o_{i_{1}}^{(t)}+o_{i_{2}}^{(t)}+\cdots+o_{i_{k}}^{(t)}\right)-\frac{k}{k+1} o_{i}^{(t)}
$$

which yields the French update rule:

$$
o_{i}^{(t+1)}=\frac{1}{k+1}\left(o_{i}^{(t)}+o_{i_{1}}^{(t)}+o_{i_{2}}^{(t)}+\cdots+o_{i_{k}}^{(t)}\right)
$$

\subsection{Topic coupling}

In the real world, topics are not independent of each other. For example, there might be good reasons that if someone changes their opinion about education then they would also change their mind about social topics in which education is a factor.

Definition 3.5. Change of opinion about topic $s_{k}$ as a result of change of opinion about topic $s_{\ell}$ is called coupling.

The coupling is done in two ways. The first case is the discrete case where there are a finite number of topics in the system. In a game the two agents can talk about some of the topics and then the coupling is performed after the interaction is complete. In the second 
model there are uncountable number of topics, at a given time step two given individuals could talk about all topics at the same time to the extent they please, i.e. they have the option of not talking about some of the topics or revealing all information about a given topic in a game, and that is captured by the so called conversation filter function. This generalized model consist of three steps. In this first phase, each opinion is changed only with the force enforced by negotiation about the given topic and in the second phase the coupling is done between topics within each individual prior to any other interactions. In the coupling phase some opinions might be pushed out of the opinion space they belong to, hence, in the third phase we would push the out of place opinions back in place.

\subsubsection{Discrete coupling}

Definition 3.6. Given a topic space $\mathbb{T}=\left\{s_{1}, s_{2}, \cdots, s_{n}\right\}$ and let $\mathbf{C}$ be the coupling matrix for the system. For two given topics $s_{\ell}$ and $s_{k}$, we define the inverse coupling strength(or inverse coupling coefficient or inverse correlation strength), denoted by $c_{\ell k}$, to be the parameter that determines how much the two topics are influenced by each other. The coefficient $c_{\ell k}$ dictates how the opinion $o\left(s_{k}\right)$ will change in response to a change in opinion $o\left(s_{\ell}\right)$ that occurs due to an interaction. $c_{\ell k}$ does not equal $c_{k \ell}$ necessarily.

A positive coupling coefficient would mean the two topics have direct correlation and a negative coefficient means the two topics have an inverse correlation. Topics are by definition completely correlated with themselves, so $c_{s_{i} s_{i}}=1$ for all topics $s_{i}$.

To derive the update rule for coupled topics, suppose that individual $i$ has their opinion changed about a topic after an interaction. This change is due to an external force (negotiation force) resulting from the opinion held by the other individual on the subject discussed during the interaction. Let $f:=1 \cdot \Delta o_{i}\left(s_{\ell}\right)$ and let topic $s_{\ell}$ to be related to topic $s_{k}$ with coefficient $c_{\ell k}$. Then the same force would be applied to opinion of the agent about subject $s_{k}$ and therefore:

$$
f=1 \cdot \Delta o_{i}\left(s_{\ell}\right)=c_{\ell k} \Delta o_{i}\left(s_{k}\right)
$$

which gives us the update rule for the opinions that are correlated to the topic $s_{\ell}$ :

$$
o_{i}^{(t+1)}\left(s_{k}\right)=o_{i}^{(t)}\left(s_{k}\right)+\frac{1}{c_{\ell k}} \Delta o_{i}^{(t)}\left(s_{\ell}\right) .
$$

\subsubsection{Continuous Kernel-based coupling}

In the previous section we modeled a discrete version of coupling where there are finite number of topics in the system and each pair of topics are coupled with their specific coupling coefficient. Here we would model the generalized continuous topic space where the coupling coefficients are determined via a two variable function and each pair of agents have the option of revealing all of their information about all topics in a single game. So, in a single time step, the two agents have the choice of talking about as many topics as they please, to whatever extent they prefer. For example, two agents can talk about their favorite food and favorite chef in an interaction, but not their favorite president. This is implemented via a conversation filter $f_{i j}(x, t) \in[0,1]$ which can evolve over time. $f_{i j}(x, t)=0$ means they 
would not talk about topic $x, f_{i j}(x, t)=1$ would mean they reveal all of their information about the given topic. It will not effect the opinions that are revealed, but it determines the weight that each revealed opinion contributes to the interaction energy.

Let $\mathbb{T}:=[0, L]$ be the topic space and $\mathcal{S}$ be the set of measurable functions $f:[0, L] \rightarrow[0,1]$. Suppose further that $\psi: \mathbb{R} \rightarrow \mathbb{R}$ is bounded. Then $o_{i} \in \mathcal{S}, \forall i \in V$. We define the interaction energy between nodes $i$ and $j$ over topic space by:

$$
Q_{i j}=\int_{0}^{L} \psi\left(\left\|o_{i}^{(t)}(x)-o_{j}^{(t)}(x)\right\|\right) f_{i j}^{(t)}(x) d x
$$

Minimizing this energy would lead us to an update rule for the agents at topic $x$.

After the conversation, each individual has updated her opinion about any given topic via interaction and then it is time to do the coupling in the topic network. In order to do this, we measure the energy between two topics for node $i$ as follows (we can have different potential energy $\phi$ for every pair of topics $x$ and $y$, hence we use the notation $\left.\phi\left(\left\|o_{i}(x)-o_{i}(y)\right\|, x, y\right)\right)$ :

$$
U_{i}[x, y]=\int_{0}^{L} \int_{0}^{L} \phi\left(\left\|o_{i}(x)-o_{i}(y)\right\|, x, y\right) k(x, y) d x d y
$$

Where the kernel $k(x, y)$ determines the connection strength between topics $x$ and $y$. To obtain the update rule for the two individuals we take the variational derivative of functional $U$.

Let

$$
U_{i}[x, y]=\int_{0}^{L} \int_{0}^{L} \phi\left(\left\|o_{i}(x)-o_{i}(y)\right\|, x, y\right) k(x, y) d x d y
$$

In ordinary calculus $\Delta f$ is computed (as a function of $x$ ) when $x$ changes by $\Delta x$, here, $\delta U$ is computed when $o_{i}$ changes by $\delta o_{i}$.

$$
\begin{aligned}
\frac{d}{d \epsilon} U_{i}[o(x) & +\epsilon h(x), o(y)]\left.\right|_{\epsilon=0} \\
& =\left.\left[\int_{0}^{L} \int_{0}^{L} \partial_{1} \phi(\|o(x)+\epsilon h(x)-o(y)\|, x, y)\left(\frac{o(x)+\epsilon h(x)-o(y)}{\|o(x)+\epsilon h(x)-o(y)\|} \cdot h(x)\right) k(x, y) d x d y\right]\right|_{\epsilon=0} \\
& =\int_{0}^{L} \int_{0}^{L} \frac{\partial}{\partial o} \phi(\|o(x)-o(y)\|, x, y)\left(\frac{o(x)-o(y)}{\|o(x)-o(y)\|} \cdot h(x)\right) k(x, y) d x d y \\
& =\int_{0}^{L}\left(\int_{0}^{L} \partial_{1} \phi(\|o(x)-o(y)\|, x, y) \frac{o(x)-o(y)}{\|o(x)-o(y)\|} k(x, y) d y\right) \cdot h(x) d x
\end{aligned}
$$

Therefore, the derivative which gives the updating rule would be:

which yields to:

$$
\frac{\delta U}{\delta o_{i}(x)}=\int_{0}^{L} \partial_{1} \phi\left(\left\|o_{i}(x)-o_{i}(y)\right\|, x, y\right) \frac{o_{i}(x)-o_{i}(y)}{\left\|o_{i}(x)-o_{i}(y)\right\|} k(x, y) d y
$$

$$
o_{i}^{(t+1)}(x)=o_{i}^{(t)}(x)-\alpha \int_{0}^{L} \partial_{1} \phi\left(\left\|o_{i}^{(t)}(x)-o_{i}^{(t)}(y)\right\|, x, y\right)\left(\frac{o_{i}^{(t)}(x)-o_{i}^{(t)}(y)}{\left\|o_{i}^{(t)}(x)-o_{i}^{(t)}(y)\right\|}\right) k(x, y) d y
$$


For the reason explained below, we break the time step into two parts, $(t, t+1 / 2)$ and $(t+1 / 2, t+1)$. Therefore, the left side of Eq. (19) is modified to $o_{i}^{(\tilde{t})}(x)$, where $\tilde{t}=t+1 / 2$.

\section{Dynamics of the opinion game}

The family of opinion game models we have introduced is rich and flexible. While the special case of symmetric interactions yields gradient flows, the full range of this model is much broader. But even in the gradient flow case, dynamics need not be simple: a sufficiently complex potential can lead to very complex dynamical behavior, at least computationally.

In this section we begin the exploration of model dynamics. Much of the section focuses on the behavior in the continuous, synchronous limit for systems in which the interaction potentials are symmetric and identical. But even in these simpler cases we find behavior that is not completely trivial.

We first study the behavior of a two agent system, each agent having a different interaction potential. This is simple enough that the behavior can be understood in detail.

Moving to three agent systems in which all potentials are the same symmetric tent function, we find that in addition to consensus clusters, there are entire neighborhoods of fixed points. For a deterministic interaction order, the discrete, asynchronous game can see subsets of these regions as fixed regions in which the system cycles endlessly. Even when the interaction order is random, these regions of fixed points can lead to slow drifting random walks in opinion space. The results from these studies are immediately relevant for systems of $N=3 k$ agents for any $k$.

We then turn to potentials that are no longer tent potentials to find non-trivial stable fixed points. These bell shaped potentials show us that even in the case of gradient systems (which is what we have when all potentials are the same) there can be interesting equilibrium states.

While our explorations in this section are admittedly just the beginning of a more comprehensive study of the model family, what we show here already suggests some richness in behavior that encourages us to push ahead with the other, more complex members of this family of models.

\subsection{Two Agent Systems}

In this section, we consider the dynamics of a two agent system:

$$
\begin{aligned}
& \dot{o}_{1}=f_{1}\left(o_{1}, o_{2}\right)=-\frac{\partial \psi_{1}\left(\left|o_{1}-o_{2}\right|\right)}{\partial \alpha_{1}} \\
& \dot{o}_{2}=f_{2}\left(o_{1}, o_{2}\right)=-\frac{\partial \psi_{2}\left(\left|o_{1}-o_{2}\right|\right)}{\partial o_{2}}
\end{aligned}
$$

The situation is simple enough that we can understand everything in detail. Two observations enable us to fairly easily unravel the dynamics of any two agent system:

1. the vector field in the 2-dimensional opinion state space, $\left.\left\{\left(o_{1}, o_{2}\right) \mid o_{1}, o_{2} \in[0,1]\right)\right\}$, is constant along $o_{1}-o_{2}=c$ lines.

2. the dynamics are completely determined by the positions of the maxima and minima of the potential functions. 
To illustrate this, we look at a couple of systems in which each individual's interaction potential is a simple tent function, though with different peak positions. We are able to give a complete picture of the simple dynamics in each case.

What about the case when the potentials are not functions only of the difference $\left|o_{1}-o_{2}\right|$ ? I.e. when

$$
\begin{aligned}
& \dot{o}_{1}=f_{1}\left(o_{1}, o_{2}\right)=-\frac{\partial \psi_{1}\left(o_{1}, o_{2}\right)}{\partial o_{1}} \\
& \dot{o}_{2}=f_{2}\left(o_{1}, o_{2}\right)=-\frac{\partial \psi_{2}\left(o_{1}, o_{2}\right)}{\partial o_{2}}
\end{aligned}
$$

While this question is mostly left to future papers, we close the section with a simple sensitivity result for the case of general potentials.

\subsubsection{Two tents}

In Fig. (3a) we see the case of two tent potentials with their tips at $\tau_{1}$ and $\tau_{2}$ where $\tau_{1} \leq \tau_{2}$, and $1-\tau_{2}=\tau_{1}$. In this simple case, the 2 dimensional state space is divided into regions in which the vector field generated by the partial derivatives of the potentials are constant. There are three cases:

1. When $o_{1}-o_{2}<\tau_{1}$, the agent's opinions move towards each other and the slope of vectors is $\frac{\Delta o_{2}}{\Delta o_{1}}=-\frac{\tau_{1}}{\tau_{2}}$.

2. If $\tau_{1}<o_{1}-o_{2}<\tau_{2}$, then, agent 1 moves away with agent two in pursuit, $\frac{\Delta o_{2}}{\Delta o_{1}}=\frac{1-\tau_{1}}{\tau_{2}}=1$.

3. Finally, if $o_{1}-o_{2}>\tau_{2}$ they repulse each other towards polarization.

We denote the region in which the opinions are attracted towards each other by $\mathrm{A}$, the region where one opinion chases the other we denote by $\mathrm{C}$, and $\mathrm{R}$ is used to label the region where they repulse each other.

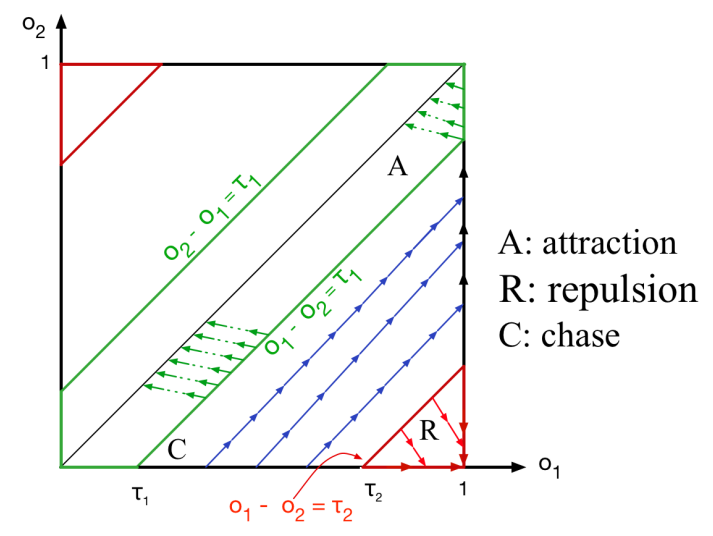

(a) $1-\tau_{2}=\tau_{1}$

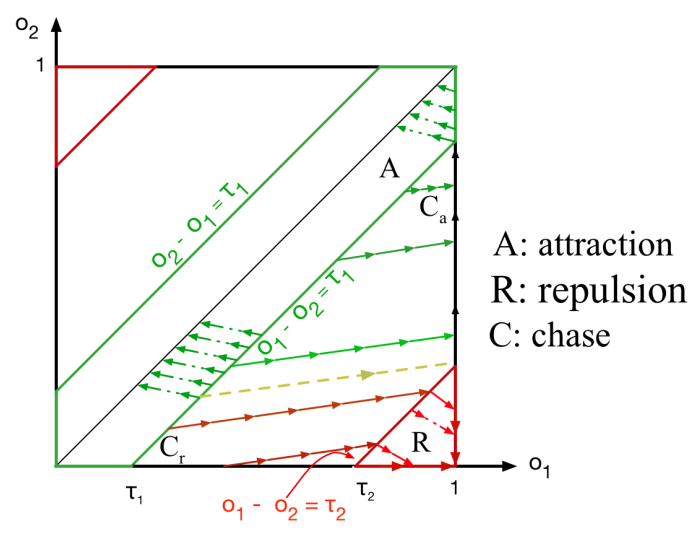

(b) $1-\tau_{2}<\tau_{1}$

Figure 3: Two agents vector fields

In the Fig. (3b) we show the case of two tent potentials with their tips at $\tau_{1}$ and $\tau_{2}$ where $\tau_{1} \leq \tau_{2}$ and $1-\tau_{2}<\tau_{1}$. Again we use $\mathrm{A}, \mathrm{C}$, and $\mathrm{R}$ for the attraction, chase and repulsion 
regions, but now the chase region is divided into 2 sub-regions, $\mathrm{C}_{a}$ and $\mathrm{C}_{r}$, depending on whether the opinion state eventually enters the attraction region or the repulsion region.

1. In region $\mathrm{A}$, the slope of vectors is $m=\Delta o_{2} / \Delta o_{1}=-\tau_{1} / \tau_{2}<-1$, directed towards consensus. All points on the line segment that connects $p=(a, a)$ to $q=(a+$ $\left.\frac{\tau_{1} \tau_{2}}{\tau_{1}+\tau_{2}}, a-\frac{\tau_{1}^{2}}{\tau_{1}+\tau_{2}}\right)$ flow towards to $p=(a, a)$.

2. We subdivide region $\mathrm{C}$ into $\mathrm{C}_{a}$ and $\mathrm{C}_{r}$ regions:

(a) The line $o_{2}=\frac{1-\tau_{1}}{\tau_{1}} o_{1}+\tau_{2}$ divides $\mathrm{C}$ into two regions.

(b) Above that line the chase eventually ends with in region $\mathrm{A}$.

(c) Below that line the chase eventually ends in region $\mathrm{R}$.

3. In the region $\mathrm{R} o_{1}-o_{2}>\tau_{2}$, the opinions repulse and $o_{1}$ converges to 1 and $o_{2}$ to 0 .

Remark 4.1. We are ignoring the fact that the tent system has undefined gradient at the tent tips, though it is not hard to deduce the results if smoothed out versions are used instead.

\subsubsection{Two Smooth Potentials}

When $\psi_{1}=\psi_{2}$ or when $\psi_{1}$ and $\psi_{2}$ are piecewise linear, the trajectories in the opinion state space are piecewise linear and the sensitivity (separation of orbits) is very simple to understand: mostly orbits that start close, remain close to each other. When there are two different potentials with non-constant derivatives, e.g. two different Gaussian-like potentials, the vector field becomes slightly more interesting, though it is still constant on $o_{1}-o_{2}=c$ lines. This again results in a system whose sensitivity to initial conditions are quite straightforward to understand. (The simple structure of the lines of unstable fixed points gives us the structure of the regions in which small perturbations result in large differences in final equilibrium positions.) When we relax the assumption that the potentials are functions of the difference $o_{1}-O_{2}$ alone, we can get more interesting behavior. In this section, we close with an example of the kind of result we can get when, instead of assuming $\psi_{1}$ and $\psi_{2}$ are functions of $\left|o_{1}-o_{2}\right|$ alone, we assume that $\psi_{1}$ and $\psi_{2}$ are smooth.

Proposition 4.1. Let $G$ be a network with two agents with smooth, compactly supported potential functions, $\psi_{1}$ and $\psi_{2}$, generating a vector field, as in Eq. (21), with Lipschitz constant $L$. Let $p=\left(o_{1}^{(0)}, o_{2}^{(0)}\right)$ be the initial opinion state of the system, and $\hat{p}=\left(\hat{o}_{1}^{(0)}, \hat{o}_{2}^{(0)}\right)$ be a perturbation of $p$. Then a bound on the difference between the states at time $T$ is given by

$$
\left\|p^{T}-\hat{p}^{T}\right\| \leq\left\|p^{(0)}-\hat{p}^{(0)}\right\| e^{T L^{*}}
$$

where $L^{*} \sim O(L)$.

Proof. Let $\psi_{1}$ and $\psi_{2}$ be smooth, compactly supported potential functions of nodes 1 and 2 respectively. The Lipschitz constant of the vector field $\left(f_{1}, f_{2}\right)$ in Eq. (21) is $L=\sqrt{L_{1}^{2}+L_{2}^{2}}$ where $L_{i}$ is Lipschitz constant of each $f_{i}$. (The partial derivatives of $\psi_{1}$ and $\psi_{2}$ are Lipschitz 
since they are continuously differentiable on a compact set.) Define $p^{(0)}=\left[\begin{array}{ll}o_{1}^{(0)} & o_{2}^{(0)}\end{array}\right]$ and $\hat{p}^{(0)}=\left[\begin{array}{ll}\hat{o}_{1}^{(0)} & \hat{o}_{2}^{(0)}\end{array}\right]$ be two initial conditions to $n$ steps of Runge-Kutta method $\left(A, b^{T}, c\right)$, using step size $h \leq h_{0}$, where $h_{0} L \rho(|A|)<1$, and let $p_{n}$ and $\hat{p}_{n}$ be the corresponding output values, then by Lemma 319A of [7] we have:

$$
\left\|p_{n}-\hat{p}_{n}\right\| \leq\left(1+h L^{*}\right)^{n}|| p_{0}-\hat{p}_{0} \|
$$

where $L^{*}=L\left|b^{T}\right|\left(I-h_{0} L|A|\right)^{-1} \mathbf{1}$. Letting $h=\frac{T}{n}$ we have

$$
\frac{\left\|p_{n}-\hat{p}_{n}\right\|}{\left\|p_{0}-\hat{p}_{0}\right\|} \leq e^{T L^{*}}
$$

Since, as $n \rightarrow \infty, p_{n} \rightarrow p^{T}$ and $\hat{p}_{n} \rightarrow \hat{p}^{T}$, this last inequality gives us the result.

Example 4.1. We begin by defining $\gamma=o_{1}-o_{2}$ and the Gaussian potential $\psi_{\mu, \sigma}(\gamma)=$ $\frac{1}{\sigma \sqrt{2 \pi}} e^{-\frac{(\gamma-\mu)^{2}}{2 \sigma^{2}}}$. Then

$$
\psi_{\mu, \sigma}^{\prime \prime}(\gamma)=\frac{1}{\sigma^{3} \sqrt{2 \pi}} e^{-\frac{(\gamma-\mu)^{2}}{2 \sigma^{2}}}\left[\frac{(\gamma-\mu)^{2}}{\sigma^{2}}-1\right]
$$

We now choose $\psi_{1}=\psi_{0.7,1}$ and $\psi_{2}=\psi_{0.3,2}$. We compute $L_{1}=\sup _{|\gamma| \leq 1}\left|\psi_{1}^{\prime \prime}(\gamma)\right|=\left|\frac{-.3992}{\sqrt{2 \pi}}\right|$ at $\gamma=0$ and $L_{2}=\sup _{|\gamma| \leq 1}\left|\psi_{2}^{\prime \prime}(\gamma)\right|=\left|\frac{-.8254}{2^{3} \sqrt{2 \pi}}\right|$ at $\gamma=1$ and consequently $L=0.164529$ and hence, $L^{*}=0.16$. As an example let $O^{(0)}=\left[\begin{array}{ll}0.2 & .1\end{array}\right]$ be an initial state and two perturbations of it, $O_{t}^{(0)}=O^{(0)}+10^{-8} \boldsymbol{v}$ and $O_{p}^{(0)}=O^{(0)}+10^{-8} \boldsymbol{w}$, where $O_{t}^{(0)}$ is perturbation in direction of tangent line to vector field at the point $\left[\begin{array}{ll}0.2 & 0.1\end{array}\right]$ and $O_{p}^{(0)}$ is perturbation in the direction perpendicular to the tangent line. $\boldsymbol{v}$ and $\boldsymbol{w}$ are unit vectors. Applying RK41 with step size of $h=0.1$ and final time $T=30$ we take $n=T / h=300$ steps to get to the points $O^{(300)}, O_{t}^{(300)}$ and $O_{p}^{(300)}$. Let $\boldsymbol{v}_{1}=O_{t}^{(0)}-O^{(0)}, \hat{\boldsymbol{v}}_{1}=O_{t}^{(300)}-O^{(300)}, \boldsymbol{w}_{1}=O_{p}^{(0)}-O^{(0)}$ and $\hat{\boldsymbol{w}}_{1}=O_{p}^{(300)}-O^{(300)}$, then we have

$$
\frac{\left\|\boldsymbol{v}_{1}\right\|}{\left\|\hat{\boldsymbol{v}}_{1}\right\|}=0.0363, \quad \frac{\left\|\boldsymbol{w}_{1}\right\|}{\left\|\hat{\boldsymbol{w}}_{1}\right\|}=1.145
$$

while by Eq. (22) we have

$$
\frac{\left\|O_{n}-\hat{O}_{n}\right\|}{\left\|O_{0}-\hat{O}_{0}\right\|} \leq(1+0.0165)^{300}=135.58
$$

showing that in this case, the bounds, though correct, are very pessimistic.

Remark 4.2. Note that in the previous example, even though the potential functions are no compactly supported in the unit square, they still have the property that all derivatives are bounded, which is all the we actually used in the previous proposition. 


\subsection{Dynamics of three agents}

\subsubsection{Regions of Fixed Points for Tent Potentials}

In this section we let $G=(V, E)$ be a a fully connected network with $|E|=3$ and study the continuous dynamical system generated by those three interacting agents. All the interaction potentials are assumed to be identical. The total energy of such a gradient system is given by:

$$
\Psi_{123}=\psi_{12}\left(\left|o_{1}-o_{2}\right|\right)+\psi_{23}\left(\left|o_{2}-o_{3}\right|\right)+\psi_{31}\left(\left|o_{3}-o_{1}\right|\right)
$$

where $\psi_{i j}$ is the potential assigned to the edge between nodes $i$ and $j$. Each interaction gives us

$$
i-j \text { interaction contribution to gradient vector field }\left\{\begin{array}{l}
\dot{o}_{i}(i, j)=-\psi_{i j}^{\prime}\left(\left|d_{i j}^{(t)}\right|\right) \frac{d_{i j}^{(t)}}{\left|d_{i j}^{(t)}\right|} \\
\dot{o}_{j}(i, j)=-\psi_{i j}^{\prime}\left(\left|d_{i j}^{(t)}\right|\right) \frac{-d_{i j}^{(t)}}{\left|d_{i j}^{(t)}\right|}
\end{array}\right.
$$

where $d_{i j}=o_{i}-o_{j}$. Along the diagonal lines in the coordinate subspace corresponding to individuals $i$ and $j$, the difference $o_{i}-o_{j}$ is constant and since both agents' behavior is enforced by the same potential, $\psi_{i j}$, the direction of movement is either $(1,-1)$ or $(-1,1)$. See Figure (4).

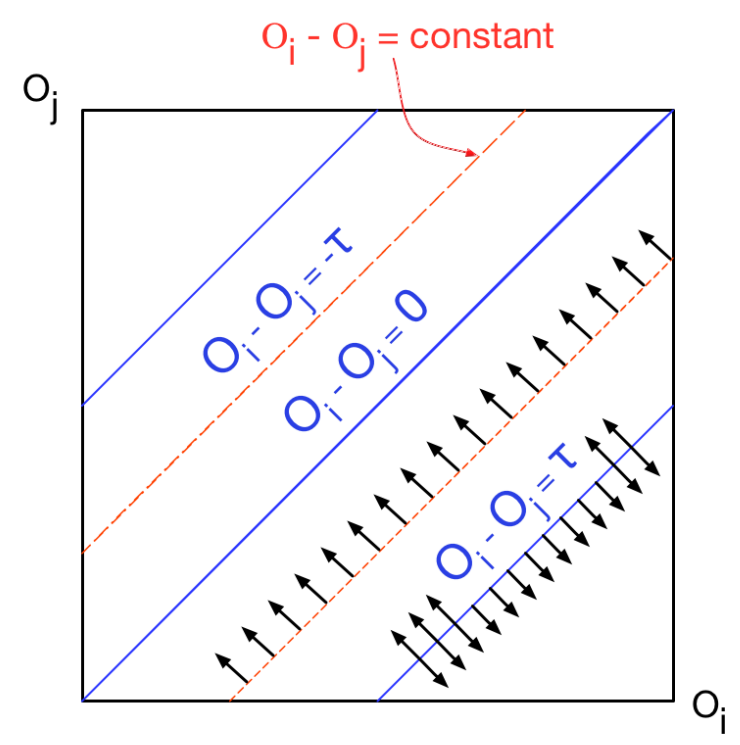

Figure 4: Coordinate subspace corresponding to individuals $i$ and $j$.

By Eq. (26) we have:

$$
\nabla \psi_{12}\left(\left|o_{2}-o_{1}\right|\right)=(a,-a, 0), \quad \nabla \psi_{13}\left(\left|o_{3}-o_{1}\right|\right)=(-b, 0, b), \quad \nabla \psi_{23}\left(\left|o_{3}-o_{2}\right|\right)=(0, c,-c)
$$

where $a, b$ and $c$ can be either positive or negative and are given by the right side of Eq. (26). Combining these, we get that the gradient vector field generated by our potential energy is 
given by:

$$
\begin{aligned}
\dot{\overrightarrow{\mathbf{o}}} & =\nabla \Psi_{123} \\
& =\nabla \psi_{12}\left(\left|o_{1}-o_{2}\right|\right)+\nabla \psi_{13}\left(\left|o_{1}-o_{3}\right|\right)+\nabla \psi_{32}\left(\left|o_{3}-o_{2}\right|\right) \\
& =(a,-a, 0)+(0, b,-b)+(-c, 0, c) \\
& =(a-c, b-a, c-b) .
\end{aligned}
$$

Equation (27) shows we will get fixed points if $a=b=c$.

Denoting the opinion state space by $\mathcal{C}_{3}=\mathbb{O}^{3}=[0,1]^{3}$, we define

$$
\mathcal{D}_{3}=\left\{\left(o_{1}, o_{2}, o_{3}\right) \mid o_{i}=c \in[0,1], 1 \leq i \leq 3\right\}
$$

to be the diagonal of $\mathcal{C}_{3}$ corresponding to consensus states, denote the set of extreme points where the system is polarized by

$$
\mathcal{E}_{3}=\{(1,0,0),(0,1,0),(0,0,1),(1,1,0),(1,0,1),(0,1,1)\} .
$$

The next theorem shows that when the common potential is a tent function, there are regions of neutral fixed points in the system that are neither consensus points nor polarization points.

Theorem 4.1. Let $G_{3}=(V, E)$ be a fully connected network of three nodes. Let all interaction potentials be the same tent potential function, with its tip at $\tau=0.5$ and maximum height $h$. Then there are subsets of $\mathcal{C}_{3} \backslash\left(\mathcal{D}_{3} \cup \mathcal{E}_{3}\right)$ with nonempty interior, which are made up of neutral fixed points.

Proof. Without loss of generality, we will consider the case $o_{1}<o_{2}<o_{3}$, other cases follows similarly. Let the point $p=\left(o_{1}, o_{2}, o_{3}\right) \in \mathcal{C}_{3}$ such that $d_{12}=o_{2}-o_{1}=\tau-\epsilon=o_{3}-o_{2}=d_{23}$ and $d_{13}=o_{3}-o_{1}=2(\tau-\epsilon)>\tau$. Since the potential function has the same slope in magnitude on both sides of $\tau=0.5$, by (27) we have:

$$
\begin{aligned}
\dot{\overrightarrow{\mathbf{o}}} & =\nabla \Psi_{123} \\
& =(\alpha h,-\alpha h, 0)+(0, \alpha h,-\alpha h)+(-\alpha h, 0, \alpha h) \\
& =(0,0,0)
\end{aligned}
$$

Moreover, the point $P$ is not an isolated fixed point. Notice that the point $p$ can be perturbed to $\hat{p}=p+\zeta(1,1,1)$ and still be a fixed point as long as $\tau<\hat{d}_{13}<1$ and $0<\hat{d}_{12}, \hat{d}_{23}<\tau$, where $\hat{d}_{i j}=\left|\hat{o}_{i}-\hat{o}_{j}\right|$.

Example 4.2. Let $G_{3}$ be a fully connected network with potential tent functions, $\tau=0.5$ and $h=1$, for each edge. Let $\left(o_{1}, o_{2}, o_{3}\right)=(0.2,0.5,0.8)$. Then each agent is pushed and pulled by the same force, $a=b=c=1$ above, by the other two agents, and therefore they do not move, hence, we have a fixed point and indeed a region of fixed points.

In the Thm (4.1) we considered only the case $o_{1}<o_{2}<o_{3}$. There are five other possible cases, and all these six regions are mutually exclusive. The regions are given in Sec. (7) and it is shown that their interior are mutually disjoint and afterward we show that there must be fixed points inside these regions.

These fixed points are not specific to three agent systems. In fact, we can immediately extend the results to the case in which there are $N=3 k$ agents. 
Corollary 4.1. Let $G=(V, E)$ be a fully connected graph with $|V|=N=3 k$ for some $k \in \mathbb{N}$ and let all the agents have the same tent potential function $\psi$ with peak at 0.5 . Then there the are subsets of $\mathcal{C}_{N} \backslash\left(\mathcal{E}_{N} \cup \mathcal{D}_{N}\right)$ with non-empty interior which are comprised of fixed points.

Proof. Let $p=\left(o_{1}, o_{2}, o_{3}\right)$ be a neutrally stable fixed point in the $R_{1}$ derived above. Moreover, let $S_{i}$ be a set of $k$ agents in the $\delta_{i}$ neighborhood of $o_{i}, i \in\{1,2,3\}$. Furthermore, let

$$
\begin{aligned}
& \max \left\{S_{2}\right\}-\min \left\{S_{1}\right\}<0.5-\epsilon \\
& \max \left\{S_{3}\right\}-\min \left\{S_{2}\right\}<0.5-\epsilon \\
& \min \left\{S_{3}\right\}-\max \left\{S_{1}\right\}>0.5+\epsilon
\end{aligned}
$$

Then since the potential function has constant and the same slope size on the whole region except $(0.5-\epsilon, 0.5+\epsilon)$, the force applied to nodes in $S_{i}$ by nodes in $S_{j}$ and nodes in $S_{k^{-}}$ $i \neq j \neq k$ - would cancel out and the only force working on nodes in the $S_{i}$ is applied by nodes in the $S_{i}$, and therefore, they all would come to consensus at a point inside the $\delta_{i}$ neighborhood of $o_{i}$, denote it by $o_{i}^{*}$ and therefore, $p^{*}=\left(o_{1}^{*}, o_{2}^{*}, o_{3}^{*}\right)$ would be a fixed point inside the $R_{1}$.

Remark 4.3. In the case of this potential $\Psi_{123}$ we know that when we are not in at a fixed point, the rate of energy decrease is bounded below, so we are guaranteed to converge to a fixed point in time less than or equal to $\frac{\sup _{\mathcal{C}_{3}} \Psi_{123}}{\alpha h}$.

Next, we look at bell-shaped potentials and the fixed points they generate using a simple example potential.

\subsubsection{Bell shaped potential}

Take a symmetric potential function about $x=0.5$ with zero slope at $x=0$ and $x=1$. We can obtain this by taking a smooth function between zero and 0.25 and then rotate, reflect and shifting it to make up the function, like Fig. (5), which corresponds to the function by Eq. (29).

$$
\psi(x)=\left\{\begin{array}{lr}
x^{2} & 0 \leq x \leq 0.25 \\
-\left(x-\frac{1}{2}\right)^{2}+\frac{1}{8} & 0.25<x<0.75 \\
(x-1)^{2} & 0.75 \leq x \leq 1
\end{array}\right.
$$

Next we show a symmetric bell-shaped potential function has lines of neutrally stable fixed points.

Theorem 4.2. Let $G=(V, E)$ be a fully connected network of 3 agents and let each agent to have the same bell-shaped potential $\psi$. Then there exist six line segments of fixed points. Moreover, these regions can be turned into unstable fixed point regions by perturbation of the potential function. 


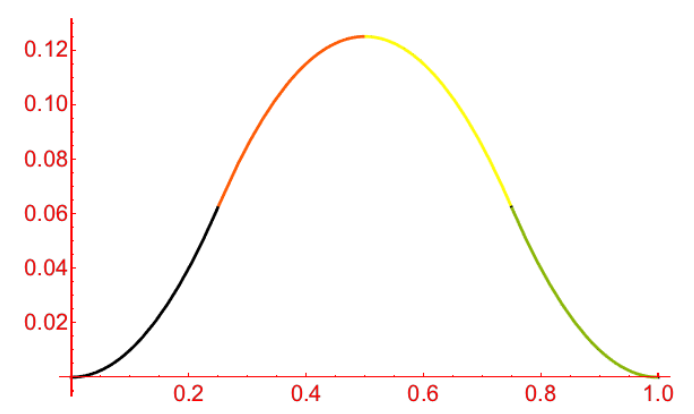

Figure 5: Potential function by rotations, reflections and shifts of $x^{2}$

Proof. Let $p=\left(o_{1}^{*}, o_{2}^{*}, o_{3}^{*}\right)$ be a given fixed point in $(\mathcal{D} \cup \mathcal{E})^{\prime}$ where $o_{1}^{*}<o_{2}^{*}<o_{3}^{*}$. Moreover, let $f_{i j}=\frac{\alpha}{2} \psi_{i j}^{\prime}$ be the force imposed by agent $i$ on agent $j$ where $\psi_{i j}^{\prime}=\psi^{\prime}\left(d_{i j}\right)$. Since $p$ is a fixed point the forces $f_{i j}$ in Fig. (6) have the same magnitude. Since $f_{21}=f_{12}$ we must have $f_{12}=f_{32}$ in order for agent two to not move. Therefore, $d_{12}=d_{23}=d \in(0.25,0.5)$ and $d_{13}=2 d \in(0.5,0.75)$. Note that $d_{12}$ and $d_{23}$ cannot be in $(0,0.25)$ because otherwise, $d_{13} \in(0,0.5)$ and there would not exist a repelling force. Since $2 d \in(0.5,0.75)$ we have to have $d \in(0.25,0.375)$.

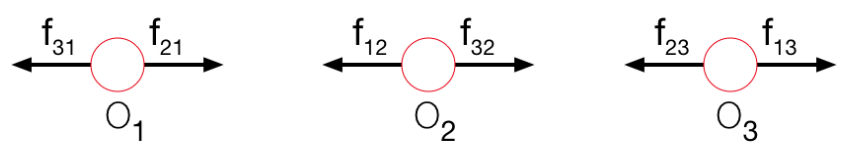

Figure 6

Starting from agent 1 , we want to have $F_{1}=f_{21}+f_{31}=0$. Since the potential function is symmetric about $x=0.5$ there exist the points $d \in(0.25,0.5)$ such that $\psi^{\prime}(d)=-\psi^{\prime}(2 d)$ and consequently $F_{1}=0$. The same is true for $F_{2}$ and $F_{3}$.

Neutrality of $p$ is shown below. In our system we have $\dot{\overrightarrow{\mathbf{o}}}=\nabla \psi_{123}$. Denote different components as follows:

$$
\left\{\begin{array}{l}
\dot{o}_{1}=f\left(o_{1}, o_{2}, o_{3}\right) \\
\dot{o}_{2}=g\left(o_{1}, o_{2}, o_{3}\right) \\
\dot{o}_{3}=h\left(o_{1}, o_{2}, o_{3}\right)
\end{array}\right.
$$

Let $p=\left(o_{1}^{*}, o_{2}^{*}, o_{3}^{*}\right)$ be a fixed point of the three-agent interactions. To show these are unstable fixed points we would use linearization of the potential about the fixed point. Let $u=o_{1}-o_{1}^{*}, v=o_{2}-o_{2}^{*}$ and $w=o_{3}-o_{3}^{*}$ be small perturbation of the fixed point. To see whether the perturbation grows or decays we have to derive differential equations for $u, v$ 
and $w$ :

$$
\begin{aligned}
\dot{u}=\dot{o}_{1} & =f\left(o_{1}^{*}+u, o_{2}^{*}+v, o_{3}^{*}+w\right) \\
& =f\left(o_{1}^{*}, o_{2}^{*}, o_{3}^{*}\right)+f_{o_{1}} u+f_{o_{3}} v+f_{o_{3}} w+\frac{1}{2 !}\left[\begin{array}{lll}
u & v & w
\end{array}\right] H\left(o_{1}, o_{2}, o_{3}\right)\left[\begin{array}{c}
u \\
v \\
w
\end{array}\right]+\cdots \\
& =f_{o_{1}} u+f_{o_{2}} v+f_{o_{3}} w+O\left(u^{2}, v^{2}, w^{2}, u v, w v, u w\right)
\end{aligned}
$$

Where $H\left(o_{1}, o_{2}, o_{3}\right)=\left[\begin{array}{lll}f_{o_{1} o_{1}} & f_{o_{1} o_{2}} & f_{o_{1} o_{3}} \\ f_{o_{2} o_{1}} & f_{o_{2} o_{2}} & f_{o_{2} o_{3}} \\ f_{o_{3} o_{1}} & f_{o_{3} o_{2}} & f_{o_{3} o_{3}}\end{array}\right]$, called the Hessian matrix and $f_{o_{i}}$ is derivative of $f$ with respect to $o_{i}$ evaluated at $\left(o_{1}^{*}, o_{2}^{*}, o_{3}^{*}\right)$. The same can be obtained for $v$ and $w$. And perturbation evolves according to

$$
\begin{aligned}
{\left[\begin{array}{c}
\dot{u} \\
\dot{v} \\
\dot{w}
\end{array}\right] } & =\left[\begin{array}{lll}
f_{o_{1}} & f_{o_{2}} & f_{o_{3}} \\
g_{o_{1}} & g_{o_{2}} & g_{o_{3}} \\
h_{o_{1}} & h_{o_{2}} & h_{o_{3}}
\end{array}\right]_{\left(o_{1}^{*}, o_{2}^{*}, o_{3}^{*}\right)}\left[\begin{array}{c}
u \\
v \\
w
\end{array}\right]+O\left(u^{2}, v^{2}, w^{2}, u v, w v, u w\right) \\
& =J\left(o_{1}^{*}, o_{2}^{*}, o_{3}^{*}\right)\left[\begin{array}{c}
u \\
v \\
w
\end{array}\right]+O\left(u^{2}, v^{2}, w^{2}, u v, w v, u w\right)
\end{aligned}
$$

which by dropping the quadratic terms we get the linearized system. Dropping the learning rate $\alpha$, we have:

$$
\begin{aligned}
\dot{o}=\nabla \Psi_{123}= & \nabla \psi_{12}+\nabla \psi_{32}+\nabla \psi_{31} \\
= & \left(\psi_{12}^{\prime}\left(\left|o_{2}-o_{1}\right|\right) \frac{o_{2}-o_{1}}{\left|o_{2}-o_{1}\right|},-\psi_{12}^{\prime}\left(\left|o_{2}-o_{1}\right|\right) \frac{o_{2}-o_{1}}{\left|o_{2}-o_{1}\right|}, 0\right)+ \\
& \left(0, \psi_{32}^{\prime}\left(\left|o_{3}-o_{2}\right|\right) \frac{o_{3}-o_{2}}{\left.\right|_{3}-o_{2} \mid},-\psi_{32}^{\prime}\left(\left|o_{3}-o_{2}\right|\right) \frac{o_{3}-o_{2}}{o_{3}-o_{2} \mid}\right)+ \\
& \left(-\psi_{31}^{\prime}\left(\left|o_{3}-o_{1}\right|\right) \frac{o_{3}-o_{1}}{\left|o_{3}-o_{1}\right|}, 0, \psi_{31}^{\prime}\left(\left|o_{3}-o_{1}\right|\right) \frac{o_{3}-o_{1}}{\left|o_{3}-o_{1}\right|}\right) \\
= & {\left[\begin{array}{l}
\psi_{12}^{\prime}\left(\left|o_{2}-o_{1}\right|\right) \frac{o_{2}-o_{1}}{\left|o_{1}-o_{1}\right|}-\psi_{31}^{\prime}\left(\left|o_{3}-o_{1}\right|\right) \frac{o_{3}-o_{1}}{o_{3}-o_{1} \mid} \\
\psi_{32}^{\prime}\left(\left|o_{3}-o_{2}\right|\right) \frac{o_{3}-o_{2}}{\left|o_{2}-o_{2}\right|}-\psi_{12}^{\prime}\left(\left|o_{2}-o_{1}\right|\right) \frac{o_{2}-o_{1}}{\left|o_{2}-o_{1}\right|} \\
\psi_{31}^{\prime}\left(\left|o_{3}-o_{1}\right|\right) \frac{o_{3}-o_{1}}{\left|o_{3}-o_{1}\right|}-\psi_{32}^{\prime}\left(\left|o_{3}-o_{2}\right|\right) \frac{o_{3}-o_{2}}{\left|o_{3}-o_{2}\right|}
\end{array}\right]^{T} } \\
= & {\left[\begin{array}{l}
f\left(o_{1}, o_{2}, o_{3}\right) \\
g\left(o_{1}, o_{2}, o_{3}\right) \\
h\left(o_{1}, o_{2}, o_{3}\right)
\end{array}\right]^{T} }
\end{aligned}
$$

Therefore, the Jacobian matrix of (32) is given by

$$
\mathbf{J}=\left[\begin{array}{ccc}
\psi_{13}^{\prime \prime}\left(\left|o_{3}-o_{1}\right|\right)-\psi_{12}^{\prime \prime}\left(\left|o_{2}-o_{1}\right|\right) & \psi_{12}^{\prime \prime}\left(\left|o_{2}-o_{1}\right|\right) & -\psi_{13}^{\prime \prime}\left(\left|o_{3}-o_{1}\right|\right) \\
\psi_{12}^{\prime \prime}\left(\left|o_{2}-o_{1}\right|\right) & -\psi_{23}^{\prime \prime}\left(\left|o_{3}-o_{2}\right|\right)-\psi_{12}^{\prime \prime}\left(\left|o_{2}-o_{1}\right|\right) & \psi_{23}^{\prime \prime}\left(\left|o_{3}-o_{2}\right|\right) \\
-\psi_{13}^{\prime \prime}\left(\left|o_{3}-o_{1}\right|\right) & \psi_{23}^{\prime \prime}\left(\left|o_{3}-o_{2}\right|\right) & \psi_{13}^{\prime \prime}\left(\left|o_{3}-o_{1}\right|\right)-\psi_{23}^{\prime \prime}\left(\left|o_{3}-o_{2}\right|\right)
\end{array}\right]
$$


Since $d_{12}=d_{32}$ the Jacobian has the following structure:

$$
\mathbf{J}=\left[\begin{array}{ccc}
b-a & a & -b \\
a & -2 a & a \\
-b & a & b-a
\end{array}\right]
$$

The Jacobian matrix is a real symmetric matrix, and therefore, the eigenvalues are real, and therefore, centers or spirals do not exist. Since there is a line of fixed points, we cannot have three dimensions all going to the fixed points. That can also be seen from the structure of the Jacobian matrix. Due to the structure of the matrix, determinant of $J$ is zero, therefore, one of the eigenvalues is zero, which indicates fixed points are not isolated. Moreover, in a real symmetric matrix, number of negative eigenvalues is the same as number of negative pivots. The Jacobian matrix $J$ given above always have two negative eigenvalues which can be easily seen by looking at pivots signs.

However, we can perturb the potential function given by Eq. (29) using non-analytical functions at the given $d_{12}, d_{13}, d_{23}$, so that the slope is kept the same, but the second derivative is changed so that the Jacobian matrix has one positive eigenvalue, one negative and a zero eigenvalue. (Two positive eigenvalues is impossible!)

We proved the theorem for $o_{1}<o_{2}<o_{3}$, the other five cases follows similarly.

Example 4.3. Let the potential function of the three agents be given by Eq. (29). The fixed points in $(\mathcal{D} \cup \mathcal{E})^{\prime}$ are of the form $p=\left(o_{1}^{*}, o_{2}^{*}, o_{3}^{*}\right)=\left(o-\frac{1}{3}, o, o+\frac{1}{3}\right)$. The line segment generated by $o$ where $o-\frac{1}{3} \geq 0$ and $o+\frac{1}{3} \leq 1$ is the line segment of neutrally stable fixed points since:

$$
F_{1}=f_{21}+f_{31}=\frac{\alpha}{2}\left(\frac{1}{3}-\frac{1}{3}\right)=0=F_{2}=F_{3}
$$

For example, if $p=(1 / 6,1 / 2,5 / 6)$, then $J\left(o_{1}^{*}, o_{3}^{*}, o_{3}^{*}\right)=\frac{1}{3}\left[\begin{array}{ccc}-2 & 1 & 1 \\ 1 & -2 & 1 \\ 1 & 1 & -2\end{array}\right]$. Eigenvalues of the Jacobian are $\sigma(\mathbf{J})=\{-3,0\}$.

Remark 4.4. Note that there are also fixed points of the forms $p=(a, a, a+0.5)$, $q=(a, a+0.5, a)$ and $r=(a+0.5, a, a)$ due to having derivative zero at end points and in the middle of the potential.

Remark 4.5. The bell shaped potential above is just one potential in a family of potentials determined by strictly increasing smooth functions $f:[0,0.25] \rightarrow \mathbb{R}^{+} \cup\{0\}$ such that $f(0)=f^{\prime}(0)=0$. The fact that the above potential generates a stable line of fixed points means that small enough perturbations of the bell shaped potential retain the non-consensus, non-polarized fixed point. Furthermore, using these bell-shaped potentials, we can obtain fixed points for systems with $N=3 k$ agents, as was done with the tent potential in Corollary 4.1. 


\subsection{Discrete game properties}

Proposition 4.2. Let $G_{3}$ be a fully connected network of three nodes and let $\psi$ be a tent potential function centered at $\tau=0.5$, with maximum $h$ and a learning rate $\alpha$. Let $p=$ $\left(\hat{o}_{1}, \hat{o}_{2}, \hat{o}_{3}\right)$ be a given point in the unit cube so that:

1. $0<d_{12}^{(0)}, d_{23}^{(0)}<0.5$

2. $d_{13}^{(0)}>0.5$

3. $\alpha h<\min \left\{0.5-d_{23}^{(0)}, \frac{d_{13}^{(0)}-0.5}{2}\right\}$ and

4. the deterministic, asynchronous game order is $(1,2) \rightarrow(2,3) \rightarrow(3,1) \rightarrow(1,2) \rightarrow \cdots$

Then $p$ and all the points in an open neighborhood of $p$ are neutrally stable in the sense that $p^{(0)}=p^{(3)}=p^{(6)}=\cdots=p^{(3 k)}=\cdots$. I.e. all points in that neighborhood define periodic orbits of with period 3.

Proof. Since the step size $\alpha h<0.5-d_{23}^{(0)}$, after node 1 playing with node 2 , we have $d_{23}^{(1)}<0.5$, and therefore, nodes 2 and 3 attract each other, and node 2 is back on its starting point. And since $\alpha h<\frac{d_{13}^{(0)}-0.5}{2}$, we have $d_{13}^{(2)}>0.5$, and therefore nodes 1 and 3 repel and they both are back on their initial positions. The loop would goes forever. Not only $p$ generates this periodic state, but also a neighbor of $p,\left(\hat{o}_{2}+\eta_{1}, \hat{o}_{2}+\eta_{2}, \hat{o}_{3}+\eta_{3}\right)$, in which the conditions on the $d_{i j}^{(0)}$ and $\alpha h$ are satisfied would be a periodic path.

Remark 4.6. The discrete, asynchronous, deterministic game will approximate the continuous synchronous game as long as we stay away from the boundaries of the 6 neutral regions: how close we can get depends on how small the learning rate is. If the step sizes in the game we are playing cause us to step across the boundary of the neutral region, then we converge to either consensus or polarization, depending on which boundary we step across. A game in which the play order is not deterministic, but is rather random, would generate a random walk that would eventually escape the neutral region.

\section{$5 \quad$ Experimental Results}

In this section we present experiments ${ }^{1}$ examining the behavior of networks whose parameters, such as population or topology or interaction potential parameters, are fixed except one. Unless otherwise is stated, the stopping condition is the window-convergence (introduced below). In these preliminary studies, we are interested in understanding how changing a parameter changes the average stabilization time and the population of the polarization/consensus clusters.

Remark 5.1. In the experiments we use the update rule $o_{i}^{(t+1)}=o_{i}^{(t)}-\frac{\alpha}{2} \psi^{\prime}\left(\left|d_{i j}\right|\right) d_{i j}$ rather than $o_{i}^{(t+1)}=o_{i}^{(t)}-\frac{\alpha}{2} \psi^{\prime}\left(\left|d_{i j}\right|\right) \frac{d_{i j}}{\left|d_{i j}\right|}$ to prevent over shooting. This might be considered as an adaptive learning rate.

\footnotetext{
${ }^{1}$ The codes for this experiments can be found here: https://github.com/HNoorazar/
} 


\subsection{Convergence and stability}

One of the primary goals of an opinion dynamics model is to understand the state of the system in the limit of an arbitrary number of time steps. Does the system reach a stable steady state, oscillate between a finite number of deterministic states, or is the outcome stochastic or unstable? In the case where multiple final states can be reached, what is the probability of any given state being reached relative to others? To reason about this we must define the concepts of convergence and stability with respect to the opinion game. Convergence relates to a slowing of change within the system, where we would say that the system has converged to a steady state if the opinion state across the entire system has stopped changing. Consensus is a special case of convergence in which the system has converged and the opinion state across the individuals is one in which every individual agrees with the others. This is the final state of the DeGroot averaging model. Our model admits converged states in which consensus is not present (e.g., reaching a final state in which opinions are split between two sub-populations). Stability relates to the sensitivity of the system to changes when it has reached a steady state. A stable system will be able to tolerate some degree of change while converged, while an unstable system may enter into a non-steady state when a change occurs and ultimately end up in a new converged final state.

\subsubsection{Definition of Window-convergence}

In order to decide when a system has converged, one could just wait until nothing changes in a sequence of interactions involving all pairs, but this is usually not a practical approach. Instead, we will use a notion of convergence (or pseudo-convergence) in which we continuously observe changes in some moving window in time and stop when some criterion has been met.

Let $\omega$ be the length of that time interval over which we monitor opinion changes. Assume there is one topic we are tracking over time. Let $E_{s}$ be a matrix of agents opinions from time $t=1$ to time $t=s$, where the $k^{t h}$ column contains the state vector of the opinions of the $N$ agents on the topic at time $k$. In this approach to a stopping criterion, we consider only the last $\omega$ columns of $E_{s}$, which we denote by $S_{s}{ }^{\prime}$ : I.e. $S_{s}=E(1: N, s-\omega+1: s)$. Define the $N$ dimensional column vector $F_{s}$ :

$$
F_{s}(i)=\max \left\{\max _{j=1, \ldots, \omega} S_{s}(i, j)-\min _{j=1, \ldots, \omega} S_{s}(i, j)-\eta, 0\right\}
$$

We stop when $\mathrm{F}$ is the zero vector.

\subsection{Effect of Initial Opinion}

In begin to see how initial opinions effect stabilization time, we generated initial opinions using normal distributions with a mean of 0.5 and a range of variances. In this case, the game was played by picking pairs at random and calculating changes in opinion for each node/agent based on the variational model determined by a tent function with peak at 0.5.

Define $\mu_{t}$ to be the mean time to stability, $\sigma_{t}$ the variance in the stabilization times, and $\mu_{p}$ to be the mean fraction of final states that are polarized (as opposed to converging 
to consensus). The following figures plot (a) initial opinion variance versus the mean and standard deviation of stabilization time, $\mu_{t}$ and $\sigma_{t}$, and (b) initial opinion variance versus the mean polarized fraction, $\mu_{p}$.

For a given $\sigma, 100$ initial opinions were drawn from the normal distribution with mean 0.5 and standard deviation $\sigma, N(0.5, \sigma)$. For every initial opinion, the experiment was run 100 times to begin to average out the effect of the random order in which the game was played.

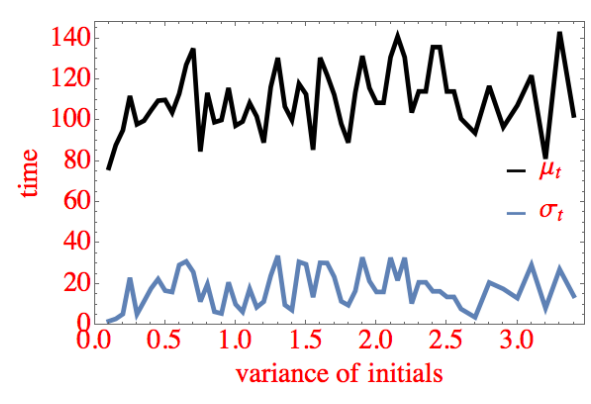

(a) Effect of initial state on stabilization time

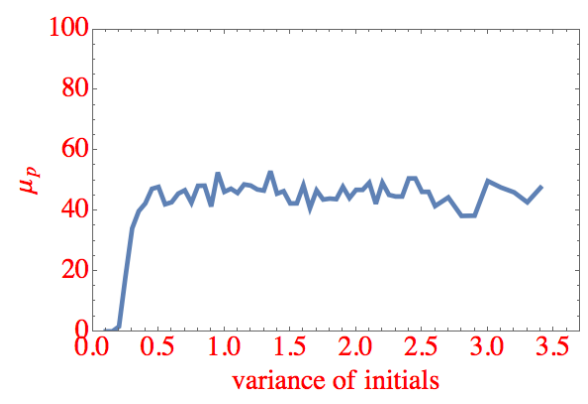

(b) Effect of initial state on polarization count

Figure 7: Initial state effects

\subsection{Effect of Tent Tip}

The experiments in this section are done with a fully connected interaction graph and a tent potential function where $\tau$, the peak of the tent, moves between 0 and 1 . Three experiments were run, each with a different strategy for picking initial conditions:

1. Step initials, in which the opinions were spread out between zero and one equally, with the same step size between them,

2. Uniform initials, in which initial opinions were sampled from the uniform distribution on $[0,1]$, and

3. Normal initials, in which initial opinions were sampled from a normal distribution. The desired number of samples were drawn from a normal distribution with mean 0 and standard deviation 1 , and then shifted and scaled so that the samples all lie within $[0,1]$.

In each of these experiments there were 20 nodes/agents were involved and for each initial condition, the game is played to convergence 1000 times.

\subsubsection{Step Initials}

Table 1 and Figure 8a show how polarization varies as the tent peak $\tau$ moves from zero to 1 . Because there is no randomness in the initial conditions, each experiment is run 1000 times 
for each position of the tent peak. This allows the effects of the randomness in the order of game negotiations to be averaged out.

Observe that we get a probability of polarization of 0.5 when $\tau \approx 0.63$. Because $\tau=0$ implies always-polarization and $\tau=1$ implies always-consensus, we might be tempted to expect that at $\tau=0.5$ we would have a probability of polarization of 0.5 . But this is a result of identifying the domain of the potential with the opinion space, even though this is not correct. The potential is a function of opinion differences, not the opinions themselves. A deeper look at this reveals that the important factors determining equilibrium states are volumes of attractors in the opinion state spaces which are controlled by the location of the tip of the tent. This is part of our ongoing work to be included in a subsequent paper to follow this one.

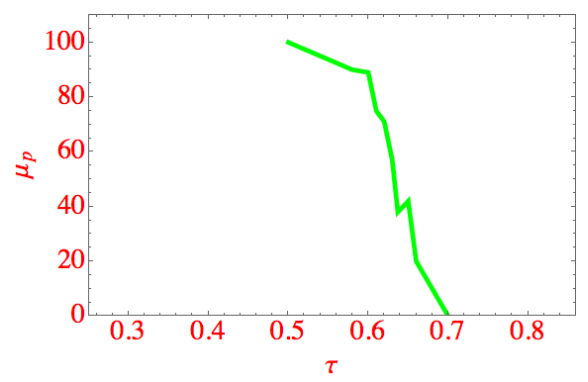

(a) Polarization mean

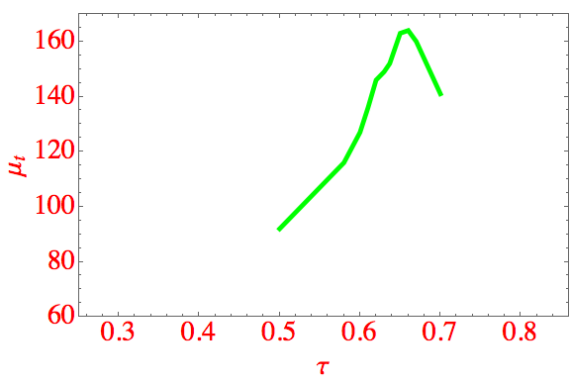

(b) Mean of stabilization time

Figure 8: Effect of moving $\tau$ on step initials.

Table 1: Step initial opinions with different tent potentials with $\tau \in[0.5,0.7]$.

\begin{tabular}{|l|l|l|l|l|l|l|l|l|l|l|l|}
\hline$\tau$ & 0.5 & 0.58 & .6 & 0.61 & 0.62 & 0.63 & 0.637 & 0.65 & 0.66 & 0.67 & .7 \\
\hline Polarization count & 100 & 90 & 89 & 75 & 71 & 57 & 38 & 42 & 20 & 15 & 0 \\
\hline Stabilization time & 92 & 116 & 127 & 136 & 146 & 149 & 152 & 163 & 164 & 160 & 141 \\
\hline
\end{tabular}

\subsubsection{Uniform initials}

In this experiment we sampled the initial opinions from a uniform distribution over $[0,1]$ 100 times, and for each sample the simulation is run 1000 times. As a result, both the order of negotiations and effect of initial sampling are taken into effect in the resulting statistics. The same is done for initial opinions sampled from normal distribution. Thus, for each value of $\tau, 100,000$ experiments are run. In Table 2 we report the normalized fraction of those experiments that resulted in polarization by dividing the total number of polarizations by 1000. 


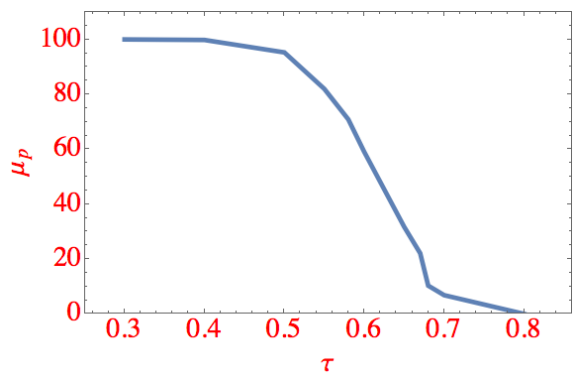

(a) Polarization mean

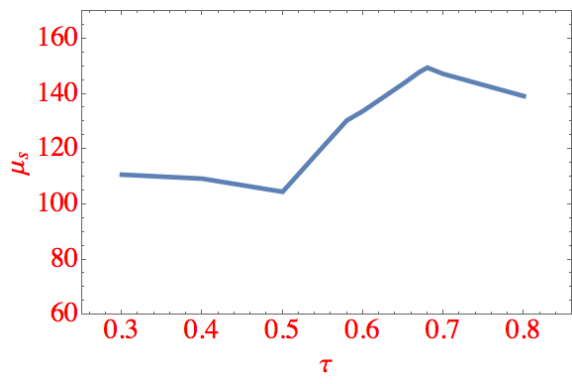

(b) Mean of stabilization time

Figure 9: Effect of $\tau$ on uniform initials

Table 2: Uniform initial opinions with different tent potentials with $\tau \in[0.3,0.8]$.

\begin{tabular}{|l|l|l|l|l|l|l|l|l|l|l|l|}
\hline$\tau$ & .3 & .4 & .5 & .55 & .58 & .6 & .65 & .67 & .68 & .7 & .8 \\
\hline$\mu_{p}$ & 100 & 99.81 & 95.27 & 81.98 & 70.87 & 59 & 31.69 & 22.08 & 10.3 & 6.82 & 0 \\
\hline$\mu_{s}$ & 110.73 & 109.29 & 104.52 & 120.75 & 130.39 & 133.76 & 143.66 & 147.84 & 149.48 & 147.20 & 139.18 \\
\hline
\end{tabular}

\subsubsection{Normal initials}

The initial 20 opinions here are generated by sampling the standard normal distribution $(\mu=0, \sigma=1)$, and then shifted and scaled so that they fit in the interval $[0,1]$.

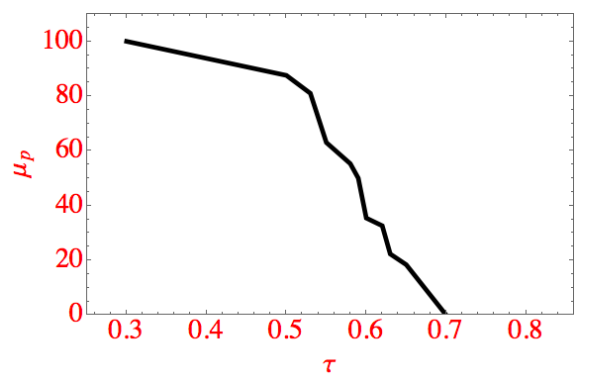

(a) Polarization mean

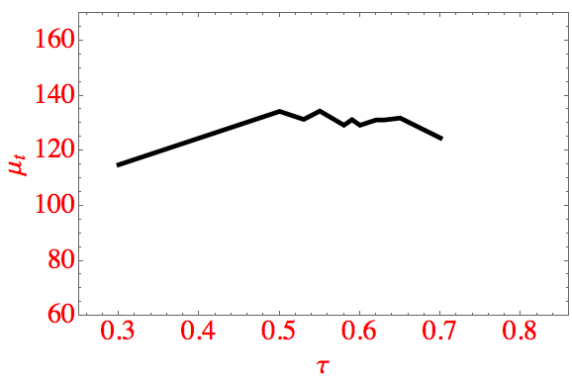

(b) Mean of stabilization time

Figure 10: Effect of $\tau$ on normal initials

Table 3: Normal initial opinions with different tent potentials with $\tau \in[0.3,0.7]$.

\begin{tabular}{|l|l|l|l|l|l|l|l|l|l|l|l|}
\hline$\tau$ & .3 & .5 & .53 & .55 & .58 & .59 & .6 & .62 & .63 & .65 & .7 \\
\hline$\mu_{p}$ & 100 & 87.57 & 81.02 & 63.04 & 55.31 & 50.02 & 35.45 & 32.63 & 22.32 & 18.41 & 0 \\
\hline$\mu_{s}$ & 114.91 & 134.27 & 131.36 & 134.39 & 129.25 & 131.26 & 129.24 & 131.15 & 131.14 & 131.85 & 124.61 \\
\hline
\end{tabular}

In Figure 11 it is clear that as the peak of the tent function increases, all experiments with different samples from different distributions show similar behavior. 
In general, one can first consider the continuous flow generated by the vector field the potentials generate and then consider the random walk behavior that results when we (essentially) compute the gradients asynchronously. These two factors: geometry of state space (determined by $\tau$ ) and random walk behavior (from random negotiation order), give rise these observed results. Because the value of $\tau$ changes the volumes of the attractors of the consensus clusters and the polarization clusters, a full explanation of these figures will wait for the next paper.

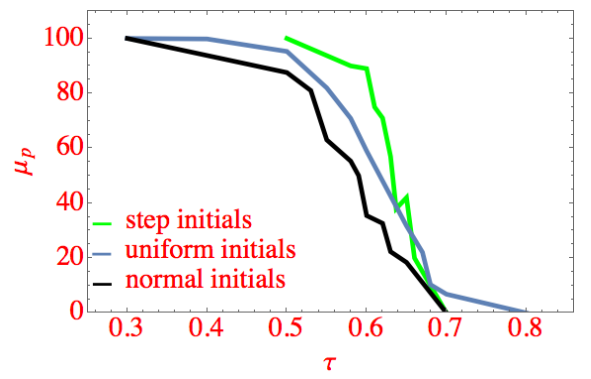

Figure 11: Polarization mean vs potential $\tau$ parameter

\subsection{Effect of Learning Rate}

In this section everything is fixed except the learning rate, which we vary to see how it effects the final state of the system and the stabilization time. In Figure 12 initial opinions and order of negotiations/conversations are the same, only the learning rate is varied. As we can see, the choice of learning rate can cause the long term equilibrium state to change from a single consensus cluster to two polarized clusters. There remains work to be performed to understand how sensitive an initial opinion state is to such effects as a function of the potential, learning rate, and distribution of initial opinion values.

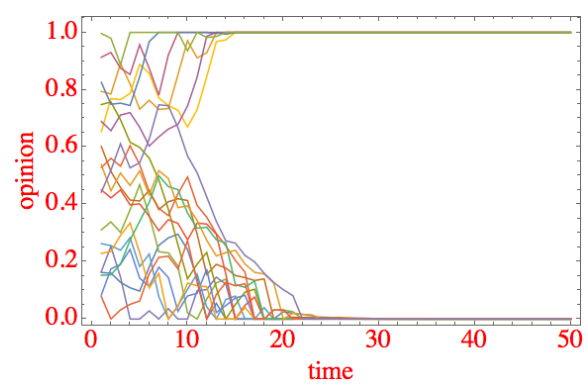

(a) $\alpha=0.2$

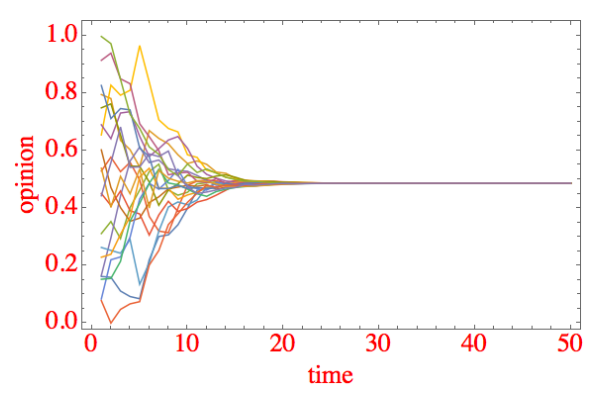

(b) $\alpha=0.3$

Figure 12: Learning rate effect

\subsection{Coupling Experiment}

In this section we take a very brief peek at the effect of coupling strength in a system with two topics and a fully connected network of 20 agents. In these 6 experiments - one each 
for 6 different coupling strengths, each time step is comprised of 10 disjoint (but random) interactions. The same set of interactions is used for each coupling strength. The experiments are terminated after 1000 time steps.

Without coupling, topic 1 polarizes and topic 2 converges to consensus. Introducing a small amount of (symmetric) coupling between the topics for all individuals, we see that the consensus state for topic 2 is destabilized. It is worth noting that there exist a small number of individuals that fail to reach a polarized state when coupling occurs. The exact cause of this behavior is the subject of future work, but we hypothesize that this is due to a small number of individuals starting with an initial opinion state with the following properties. For topic 1, they start with an opinion that leads to the consensus state with a set of other individuals. On topic 2, since everyone moves to consensus there exists only one cluster in equilibrium. When coupling is enabled, the topic 2 consensus state is perturbed and polarization occurs. In the case of the individuals who oscillate over the long term, they likely are in a situation where their opinion on topic 2 polarizes with the opposite set of individuals than they cluster with on topic 1. As such, they experience a constant tug from each population towards the opposing polarized state and cannot reach and stay at the polarized opinion state for either topic.
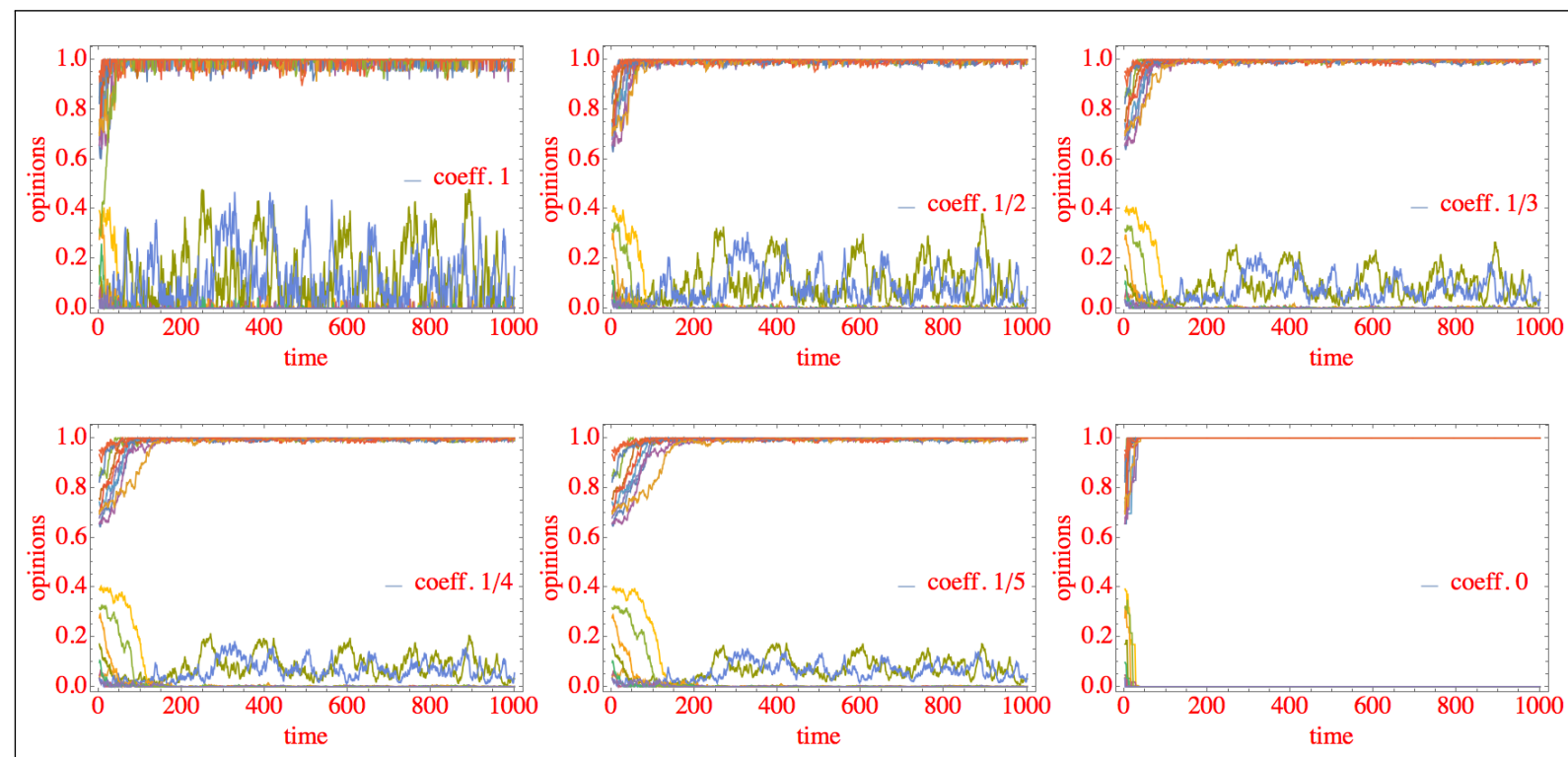

Figure 13: Different coupling coefficients - topic 1

\section{Conclusions and future work}

The work presented in this paper introduces a potential based model with inter-topic coupling. As defined, the model is relatively general as we show by embedding other models from the literature in our framework. There remain a large number of questions that can be studied based on this work. We will provide a few noteworthy questions that we identified while performing this research. First, a number of recent publications have studied 

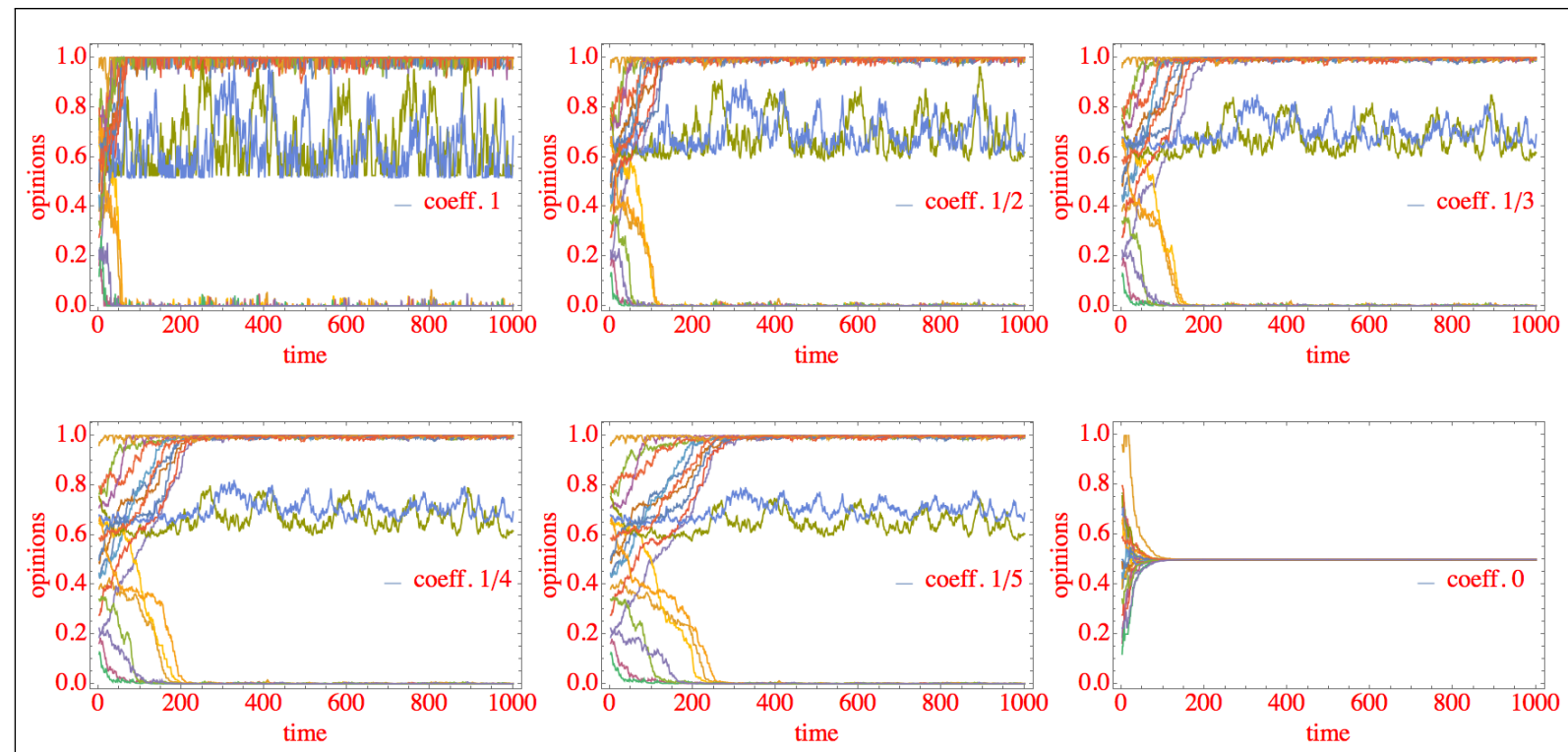

Figure 14: Different coupling coefficients - topic 2

the impact of network properties (e.g., centrality measures) on dynamical systems that are dependent on the network. Furthermore, it is known that real-world systems that are best represented by a network are often not static and instead exhibit time-varying properties both in connectivity as well as parameters such as edge weights.

As mentioned in places within the paper, we have chosen specific update criteria for modeling single agent pairs interacting and sharing opinions. Other update methods can be created that impose differing levels of synchronous behavior, differing subpopulations, and so on. These will likely have an effect on long term behavior of the model and should be studied. One of the more interesting initial results that we are studying in more detail is the cross-over point shown in Fig. (9a). Why do we observe the 50/50 polarization/consensus split occur when the tent $\tau$ parameter is approximately 0.6? Can this value be predicted analytically from the update rules, potential function, and model parameters?

A number of questions can be posed about the long term evolution of the model. We can study more about dynamics of the continuous system such as determining the conditions under which consensus/polarization occurs. Determining a lower bound on convergence time would be another interesting question. In particular, we would like to understand the long term state reached when individuals oscillate due to coupling of opinions that disagree with larger subpopulations - does this oscillation run indefinitely, or does it damp out and eventually move to a polarized state? Many of parameters of the models can also be made dynamic. For example, we may allow coupling or inter-agent weights to deviate from some equilibrium state to represent transient phenomena (e.g., conflicts).

We believe that this work represents a noteworthy accomplishment in opinion dynamics research for two reasons: it provides a flexible framework for exploring variant models based on a common core, and by adopting a common framework we are able to then use theorems about one model to reason about others. The common model framework will allow such 
translation of theorems and properties from one model to another, shedding light on models that would be difficult to analyze directly.

\section{Appendix}

Here we will show how regions of fixed points look like assuming each person or each edge has a tent potential function assigned to it with $\tau=0.5$. The regions are given by:

- $R_{1}=\left\{0<o_{2}-o_{1}<\tau\right\} \cap\left\{0<o_{3}-o_{2}<\tau\right\} \cap\left\{\tau<o_{3}-o_{1} \leq 1\right\}$, where $o_{1}<o_{2}<o_{3}$.

- $R_{2}=\left\{0<o_{1}-o_{2}<\tau\right\} \cap\left\{0<o_{3}-o_{1}<\tau\right\} \cap\left\{\tau<o_{3}-o_{2} \leq 1\right\}$, where $o_{2}<o_{1}<o_{3}$.

- $R_{3}=\left\{0<o_{3}-o_{1}<\tau\right\} \cap\left\{0<o_{2}-o_{3}<\tau\right\} \cap\left\{\tau<o_{2}-o_{1} \leq 1\right\}$, where $o_{1}<o_{3}<o_{2}$.

- $R_{4}=\left\{0<o_{3}-o_{2}<\tau\right\} \cap\left\{0<o_{1}-o_{3}<\tau\right\} \cap\left\{\tau<o_{1}-o_{2} \leq 1\right\}$, where $o_{2}<o_{3}<o_{1}$.

- $R_{5}=\left\{0<o_{1}-o_{3}<\tau\right\} \cap\left\{0<o_{2}-o_{1}<\tau\right\} \cap\left\{\tau<o_{2}-o_{3} \leq 1\right\}$, where $o_{3}<o_{1}<o_{2}$.

- $R_{6}=\left\{0<o_{2}-o_{3}<\tau\right\} \cap\left\{0<o_{1}-o_{2}<\tau\right\} \cap\left\{\tau<o_{1}-o_{3} \leq 1\right\}$, where $o_{3}<o_{2}<o_{1}$.

Note that in the first two cases, for $R_{1}$ and $R_{2}$, the condition $o_{1}<o_{2}$ and $o_{2}<o_{1}$ proves that $R_{1} \cap R_{2}=\varnothing$. Similarly, $R_{i} \cap R_{j}=\varnothing, 1 \leq i, j \leq 6$.

In the first case we have

$$
R_{1}=\left\{0<o_{2}-o_{1}<\tau\right\} \cap\left\{0<o_{3}-o_{2}<\tau\right\} \cap\left\{\tau<o_{3}-o_{1} \leq 1\right\}
$$

The first part of this region would give us two planes given by

$$
P 1 \equiv o_{2}-o_{1}=0 \text { and } P 2 \equiv o_{2}-o_{1}=\tau
$$

This region is drawn in Fig. (15a). And of course there are the two boundary planes, the cube walls $o_{1}=0$ and $o_{2}=1$, which we do not mention it, because the unit cube is the whole space we are existing in, but we would not forget about them. The planes given by Eq. (34) is intersected by the two planes from the second region given by:

$$
P 3 \equiv o_{3}-o_{2}=0 \text { and } P 4 \equiv o_{3}-o_{2}=\tau
$$

drawn in Fig. (15b).

Therefore, $P 1$ intersects $P 3$ in a line $L_{13}$ and $P 4$ in a line $L_{14}$, and the same happens for P2.

The normal vector of $P 1$ and $P 2$ is $\mathbf{n}_{\mathbf{1}}=\mathbf{n}_{\mathbf{2}}=\left[\begin{array}{lll}-1 & 1 & 0\end{array}\right]$, and we also have $\mathbf{n}_{\mathbf{3}}=\mathbf{n}_{\mathbf{4}}=$ $\left[\begin{array}{lll}0 & -1 & 1\end{array}\right]$. Hence, the direction of $L_{13}, L_{14}, L_{23}$ and $L_{24}$ all are the same and is given by

$$
\mathbf{v}=\mathbf{n}_{1} \times \mathbf{n}_{3}=\left[\begin{array}{lll}
1 & 1 & 1
\end{array}\right]=\mathbf{n}_{1} \times \mathbf{n}_{4}=\mathbf{n}_{2} \times \mathbf{n}_{3}=\mathbf{n}_{2} \times \mathbf{n}_{4}
$$




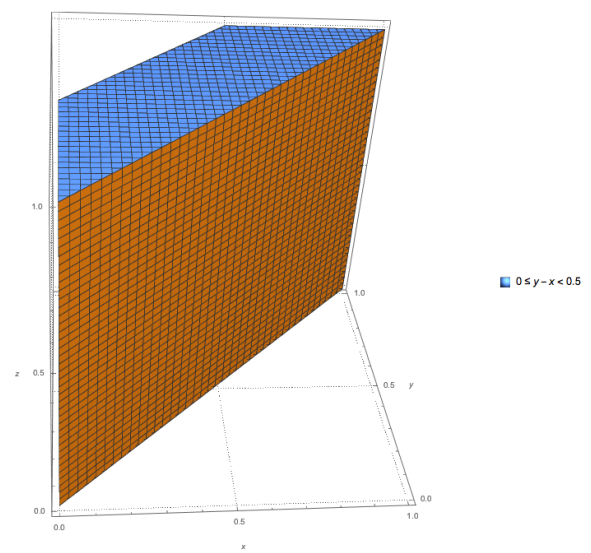

(a) First Part of $R_{1}$

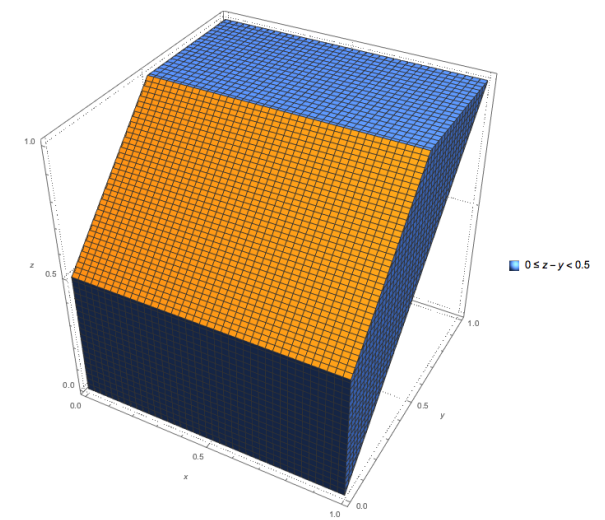

(b) Second Part of $R_{1}$

Figure 15: First two parts of $R_{1}$

The line $L_{13}$ is given by

$$
\left(o_{1}, o_{2}, o_{3}\right)=t(1,1,1)
$$

and $L_{14}$ is given by

$$
\left(o_{1}, o_{2}, o_{3}\right)=(0,0, \tau)+t(1,1,1)
$$

the lines $L_{23}$ and $L_{24}$ are given below respectively:

$$
\begin{gathered}
\left(o_{1}, o_{2}, o_{3}\right)=(0, \tau, \tau)+t(1,1,1) \\
\left(o_{1}, o_{2}, o_{3}\right)=(0, \tau, 2 \tau)+t(1,1,1)
\end{gathered}
$$

So, the area surrounded by the first two parts of $R_{1}$ is given by the four planes given by Eq. (34) and Eq.(35), borders are given by (36) - (39) and the surfaces of the unit cube. (Fig. 16)

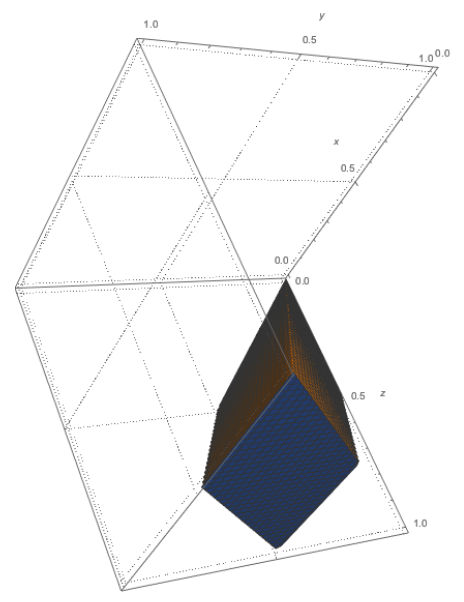

Figure 16: First two parts intersection 
This area is cut by two other planes from the third part of defining $R_{1},\left\{\tau<o_{3}-o_{1} \leq 1\right\}$.

$$
P 5 \equiv o_{3}-o_{1}=\tau \text { and } P 6 \equiv o_{3}-o_{1}=1
$$

Other than the cube's surfaces, the box has 4 sides inside the cube. Let's find the intersection of $P 5$ with those 4 sides of the box. We know

$$
\mathbf{n}_{1}=\mathbf{n}_{2}=\left[\begin{array}{lll}
-1 & 1 & 0
\end{array}\right], \mathbf{n}_{\mathbf{3}}=\mathbf{n}_{\mathbf{4}}=\left[\begin{array}{lll}
0 & -1 & 1
\end{array}\right] \text { and } \mathbf{n}_{\mathbf{5}}=\left[\begin{array}{lll}
-1 & 0 & 1
\end{array}\right]
$$

Consequently,

$$
\mathbf{n}_{\mathbf{1}} \times \mathbf{n}_{\mathbf{5}}=\left[\begin{array}{lll}
1 & 1 & 1
\end{array}\right] \text { and } \mathbf{n}_{\mathbf{3}} \times \mathbf{n}_{\mathbf{5}}=\left[\begin{array}{lll}
-1 & -1 & -1
\end{array}\right]
$$

and we get:

$$
\left\{\begin{array}{l}
L_{15} \equiv(0,0, \tau)+t(1,1,1) \\
L_{25} \equiv(0, \tau, \tau)+t(1,1,1) \\
L_{35} \equiv(-\tau, 0,0)+t(1,1,1) \\
L_{45} \equiv(0,0, \tau)+t(1,1,1)
\end{array}\right.
$$

Hence, the region is given by:

$$
\left\{\begin{array}{llll}
P 1 \text { from } L_{13} & \text { to } & L_{14} \\
P 2 \text { from } L_{23} & \text { to } & L_{24} \\
P 3 \text { from } L_{13} & \text { to } & L_{23} \\
P 4 \text { from } & L_{14} & \text { to } & L_{24}
\end{array}\right.
$$

This box is cut by the fifth plane, $o_{3}-o_{1}=\tau$. Observe that $L_{14}=L_{15}=L_{45}$ and $L_{23}=L_{25}=L_{35}$. The new box has five sides to it of which two are the unit cube walls, and the other three are P5, P2 and P4. (Fig. 17)

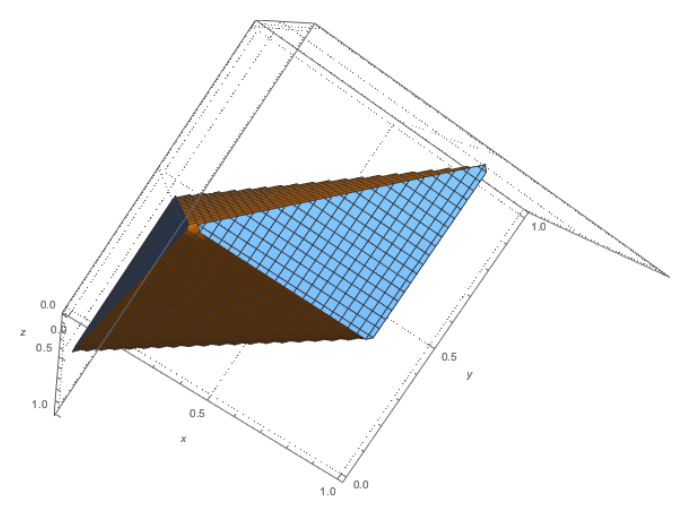

(a) $R_{1}$-Angle One

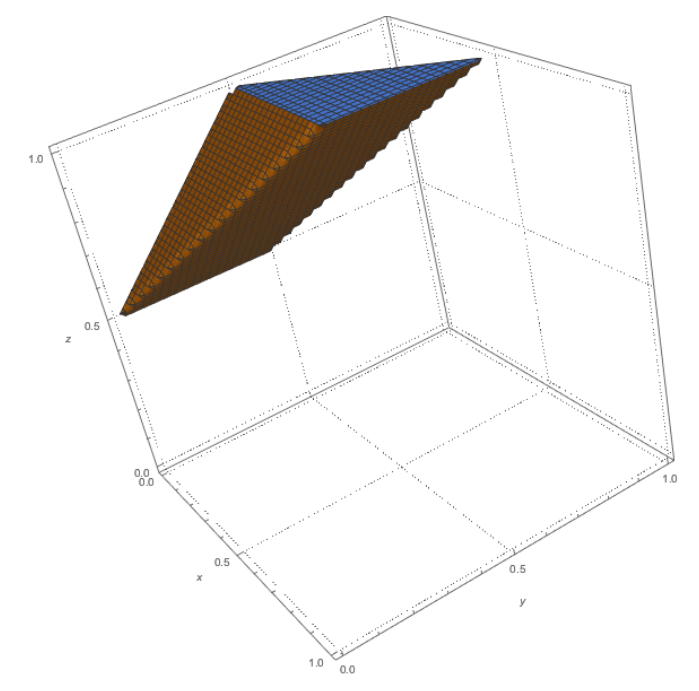

(b) $R_{1}$-Angle Two

Figure 17: The $R_{1}$ region 


\section{References}

[1] C. Altafini. Consensus problems on networks with antagonistic interactions. IEEE Transactions on Automatic Control, 58(4):935-946, April 2013.

[2] R. Axelrod. The Dissemination of Culture: A Model with Local Convergence and Global Polarization. Journal of Conflict Resolution, 41(2):203-226, 1997.

[3] Andrea Baronchelli. The minimal Naming Game : a complex systems approach. pages $1-24,2010$.

[4] Andrea Baronchelli, Tao Gong, Andrea Puglisi, and Vittorio Loreto. Modeling the emergence of universality in color naming patterns. Proceedings of the National Academy of Sciences of the United States of America, 107(6):2403-2407, 2010.

[5] Soham Biswas and Parongama Sen. Model of binary opinion dynamics: Coarsening and effect of disorder. Physical Review E - Statistical, Nonlinear, and Soft Matter Physics, $80(2): 4-7,2009$.

[6] V. D. Blondel, J. M. Hendrickx, and J. N. Tsitsiklis. On krause's multi-agent consensus model with state-dependent connectivity. IEEE Transactions on Automatic Control, 54(11):2586-2597, Nov 2009.

[7] John C Butcher. Numerical methods for ordinary differential equations. 2008.

[8] Guillaume Deffuant, David Neau, Frederic Amblard, and Gérard Weisbuch. Mixing beliefs among interacting agents. Advances in Complex Systems, 03(01n04):87-98, 2000.

[9] Morris DeGroot. Reaching a Consensus. Journal of the American Statistical Association, 69(345):118-121, 1974.

[10] Fei Ding, Yun Liu, Bo Shen, and Xia-Meng Si. An evolutionary game theory model of binary opinion formation. Physica A: Statistical Mechanics and its Applications, 389(8):1745-1752, 2010.

[11] Jan Christian Dittmer. Consensus formation under bounded confidence. Nonlinear Analysis: Theory, Methods Eramp; Applications, 47(7):4615-4621, 2001.

[12] Emile Durkheim. The division of labour in society. New York: The Free Press, 1893.

[13] Andreas Flache and Michael W. Macy. Small Worlds and Cultural Polarization. The Journal of Mathematical Sociology, 35(May 2015):146-176, 2011.

[14] Follmer, Hans. Random economies with many interacting agents. Journal of Mathematical Economics, 1(1):51-62, March 1974.

[15] Santo Fortunato, Vito Latora, Alessandro Pluchino, and Andrea Rapisarda. Vector Opinion Dynamics in a Bounded Confidence Consensus Model. International Journal of Modern Physics C, 2005. 
[16] J R French. A formal theory of social power. Psychological review, 63(3):181-194, 1956.

[17] N. E. Friedkin. A structural theory of social influence. Cambridge Univ. Press, 1998.

[18] N. E. Friedkin. A Formal Theory of Reflected Appraisals in the Evolution of Power. Administrative Science Quarterly, 56(4):501-529, 2011.

[19] N. E. Friedkin and E. Johnsen. Social influence networks and opinion change. Advances in Group Processes, 16:1-29, 1999.

[20] N. E. Friedkin and Eugene C. Johnsen. Social influence network theory. Cambridge Univ. Press, 2011.

[21] S. Galam. Local dynamics vs. social mechanisms: A unifying frame. EPL (Europhysics Letters), 70(6), 2005.

[22] S. Galam and S. Moscovici. Towards a Theory of Collective Phenomena - Consensus and Attitude Changes in Groups. European Journal of Social Psychology, 21(1):49-74, 1991.

[23] Rainer Hegselmann and Ulrich Krause. Opinion Dynamics and Bounded Confidence: Models, Analysis and Simulation. Journal of Artificial Societies and Social Simulation, $5(3), 2002$.

[24] Peng Jia, Anahita Mirtabatabaei, N. E. Friedkin, and Francesco Bullo. Opinion Dynamics and the Evolution of Social Power in Influence Networks. SIAM Review, 57(3):367$397,2015$.

[25] P. Kay. Resolving the question of color naming universals. Proceedings of the National Academy of Sciences, 100(15):9085-9089, 2003.

[26] Oussama Khatib. Real time obstacle avoidance for manipulators and mobile robots, 1986.

[27] U Krause. A Discrete Nonlinear and Non-Autonomous Model of Consensus Formation. In Communications in Difference Equations. CRC Press, 2000.

[28] L. Li, A. Scaglione, A. Swami, and Q. Zhao. Consensus, polarization and clustering of opinions in social networks. IEEE Journal on Selected Areas in Communications, 31(6):1072-1083, 2013.

[29] J Lorenz. Continuous Opinion Dynamics Under Bounded Confidence: A Survey. International Journal of Modern Physics C, 18(12):1819, 2007.

[30] Jan Lorenz. A stabilization theorem for dynamics of continuous opinions. Physica A: Statistical Mechanics and its Applications, 355(1):217-223, 2005.

[31] Andre C R Martins. Continuous Opinions and Discrete Actions in Opinion Dynamics Problems. International Journal of Modern Physics C, 19(4), 2007. 
[32] Michael Mäs, Andreas Flache, and Dirk Helbing. Individualization as Driving Force of Clustering Phenomena in Humans. PLoS Computational Biology, 6(10):e1000959, 2010.

[33] Anahita Mirtabatabaei and Francesco Bullo. On opinion dynamics in heterogeneous networks. American Control Conference, 2011.

[34] A. Nedić and B. Touri. Multi-dimensional hegselmann-krause dynamics. 2012 IEEE 51st IEEE Conference on Decision and Control (CDC), pages 68-73, 2012.

[35] Sergey E Parsegov, Anton V Proskurnikov, Roberto Tempo, and N. E. Friedkin. A Novel Multidimensional Model of Opinion Dynamics in Social Networks. 2015.

[36] A. V. Proskurnikov, A. S. Matveev, and M. Cao. Opinion dynamics in social networks with hostile camps: Consensus vs. polarization. IEEE Transactions on Automatic Control, 61(6):1524-1536, June 2016.

[37] Andrea Puglisi, Andrea Baronchelli, and Vittorio Loreto. Cultural route to the emergence of linguistic categories. Proceedings of the National Academy of Sciences of the United States of America, 105(23):7936-7940, 2008.

[38] J H Reif and H Wang. Social potential fields: a distributed behavioral control for autonomous robots. Robotics \& Autonomous Systems, 27(3):171-194, 1999.

[39] Craig W. Reynolds. Flocks, herds and schools: A distributed behavioral model. ACM SIGGRAPH Computer Graphics, 21(4):25-34, 1987.

[40] Dietrich Stauffer. How to Convince Others? Monte Carlo Simulations of the Sznajd Model. AIP Conference Proceedings, 690:147-155, 2003.

[41] Dietrich Stauffer, A. O. Sousa, and C. Schulze. Discretized opinion dynamics of Deffuant on scale-free networks. Journal of Artificial Societies and Social Simulation, 7(3):21, 2003.

[42] Katarzyna Sznajd-Weron and Józef Sznajd. Opinion evolution in closed community. International Journal of Modern Physics C, 11(06):1157-1165, 2000.

[43] G. Weisbuch. Bounded confidence and social networks. The European Physical Journal B, 38(2):339-343, 2004.

[44] G Weisbuch, G Deffuant, F Amblard, and J P Nadal. Meet, discuss, and segregate! Complexity, 7(3):55-63, 2002. 